\title{
A nonlinear elastic approach to modelling the hydro-mechanical behaviour of the SEALEX experiments on compacted MX-80 bentonite
}

\author{
A. P. Fraser Harris ${ }^{1}$ (D) C. I. McDermott ${ }^{1}$ - A. E. Bond ${ }^{2} \cdot$ K. Thatcher ${ }^{2}$. \\ S. Norris ${ }^{3}$
}

Received: 29 February 2016/ Accepted: 28 October 2016/Published online: 14 November 2016

(c) The Author(s) 2016. This article is published with open access at Springerlink.com

\begin{abstract}
Hydraulic seals using compacted sand-bentonite blocks are an important part of the closure phase of deep geological disposal facilities for the isolation of many categories of radioactive wastes. An understanding of the hydromechanical behaviour of these seals and the ability to model their behaviour is a key contribution to safety cases and licence applications. This work reports the development of a hydro-mechanically coupled model and its application to the simulation of a range of test conditions investigated in the SEALEX experiments conducted by IRSN at Tournemire URL. The work has been conducted as part of the recently completed DECOVALEX-2015 project. Richards' equation for unsaturated fluid flow is coupled to a nonlinear elastic strain-dependent mechanical model that incorporates a moving finite element mesh, and calibrated against laboratory experiments. Stress and volumetric dependencies of the water retention behaviour are incorporated through the Dueck suction concept extended to take into account permanent changes in water retention behaviour during consolidation. Plastic collapse in laboratory results is modelled with the application of a source term activated by a threshold defined in terms of the net axial stress and net suction. The model is used to simulate both a $1 / 10$ scale mock-up
\end{abstract}

This article is part of a Topical Collection in Environmental Earth Sciences on "DECOVALEX 2015", guest edited by Jens T.

Birkholzer, Alexander E. Bond, John A. Hudson, Lanru Jing, Hua Shao and Olaf Kolditz.

$\triangle$ A. P. Fraser Harris

a.p.fraser-harris@ed.ac.uk

1 The University of Edinburgh, Edinburgh, UK

2 Quintessa Ltd, Henly-on-Thames, UK

3 Radioactive Waste Management Limited, Harwell, Oxfordshire, UK laboratory test and full-scale in situ performance test and is capable of reproducing the major trends in the data with just nine mechanical parameters and an experimentally defined stress threshold.

Keywords Hydro-mechanical (HM) coupled processes . Bentonite $\cdot$ Numerical modelling $\cdot$ Radioactive waste disposal

\section{Introduction}

Deep geological disposal facilities (GDF) comprising engineered and geological barriers are internationally considered as the most feasible option for long-term isolation and disposal of radioactive wastes (Kim et al. 2011). Regional groundwater flow could provide a mechanism for radionuclides to be transported from disposed waste to the biosphere, so it is fundamental to many disposal concepts that a GDF is effectively sealed. Hydraulic seals, designed to perform this function in current research and development programmes, are primarily composed of unsaturated compacted blocks of bentonite (Andra 2005). This is an expansive clay that swells to fill engineered voids on exposure to water, and has low permeability to ensure diffusion-dominated flow and advantageous sorptive properties to inhibit radionuclide migration (Hansen et al. 2013). The specific role of bentonite in a GDF may vary from concept to concept, e.g. in addition to hydraulic seals, bentonite is also proposed as a buffer material around high-level waste canisters (e.g. Oy 2010) and as a backfill material for excavation drifts (e.g. NAGRA 2009; Oy 2010).

In order to support safety case and licence applications, a thorough understanding of bentonite behaviour and predictive capability is required. The engineering lifetime of a 
GDF is expected to be in excess of 100,000 years, and, as such, numerical modelling is required in order to provide confidence in the safety performance of a hydraulic seal. Therefore, development of numerical models for the simulation and prediction of bentonite behaviour under the range of conditions relevant to its application within a GDF is an active area of research (Navarro et al. 2014, 2015; Saba et al. 2014a, b; Ferrari and Seiphoori 2015).

Numerical models of unsaturated bentonite behaviour under a range of conditions typically couple Richards' equation or multiphase flow to a chosen mechanical model, such as nonlinear elasticity (Fredlund and Rahardjo 1993; Cui et al. 2002) or elasto-plasticity (Gens and Alonso 1992; Wheeler and Sivakumar 1995). This is achieved through the dependence of hydraulic parameters on the mechanical stresses and has led to the extension of mechanical elastoplastic models such as the Cam Clay and Mohr-Coulomb models to include the dependence of soil suction or saturation in the mechanical formulation (Gens and Alonso 1992; Börgesson et al. 1995; Wheeler and Sivakumar 1995; Alonso and Vaunat 1999; Sánchez et al. 2005; Navarro et al. 2014; Zhou and Sheng 2015).

In recent years, elasto-plastic models, such as the Barcelona Basic Model (BBM) or the Barcelona Expansive Model (BExM), have become predominant in the simulation of unsaturated soils and expansive materials (Alonso et al. 1990; Gens and Alonso 1992; Alonso and Vaunat 1999; Navarro et al. 2014). Elasto-plastic approaches based on different combinations of stress variables (e.g. Wheeler and Sivakumar 1995) and generalised plasticity models addressing the double structure of bentonite have also been proposed (Sánchez et al. 2005). The BBM and BExM are extensions of the modified Cam Clay model that incorporate soil suction in the mechanical solution through an additionally defined failure surface, known as the loadingcollapse curve (Alonso et al. 1990; Gens and Alonso 1992; Alonso and Vaunat 1999). The BExM is an extension of the BBM in which the effect of the micro- and macrostructure of bentonite on the mechanical behaviour has been addressed by additional fitting functions (Gens and Alonso 1992; Alonso and Vaunat 1999). The effect of the double structure on hydraulic behaviour has also recently been included in these formulations through parameterisation of two water retention curves for each structural scale (Alonso et al. 2011; Navarro et al. 2015).

However, the increasing complexity of the models has led to increasing numbers of parameters used for calibration. For example, parameterisation of the BBM for a constant volume test in Navarro et al. (2015) requires 14 mechanical parameters, and Rutqvist et al. (2011) report 18 mechanical parameters in the implementation of the BBM in TOUGH-FLAC. While this provides flexibility in reproducing experimental data, the final parameterisation may not be unique and may require an extensive experimental programme to calibrate the model to. Consequently, the predictive potential of models with large parameter sets depends on the completeness of the calibration data set.

In this paper, we present a comparatively simple model based on nonlinear elasticity coupled to Richards' equation for unsaturated fluid flow. The motivation of this study is to investigate an alternative method to that currently used, in an effort to minimise the parameter set required to simulate the general hydro-mechanical behaviour of bentonite. Nonlinear mechanical behaviour is simulated with an incremental function in which the mechanical properties of the bentonite are dependent on the strain history of the sample. An Updated Lagrangian finite element mesh is developed within the open source code OpenGeoSys (Kolditz et al. 2016) for use within the mechanical solution. Wetting-induced collapse is modelled through an applied load activated when a threshold defined in terms of axial stress and net suction is exceeded. Water retention behaviour in unsaturated expansive soils has been shown to be both stress and volume dependent (Gallipoli et al. 2003; Dueck 2004; Dueck and Börgesson 2007). A simple extension to the method proposed by Dueck and Börgesson (2007) has been implemented in this model to complete the hydro-mechanical coupling.

The model described in this paper has been developed as part of the DECOVALEX-2015 project (Bond et al. $2014 \mathrm{a}, 2015 \mathrm{a}, \mathrm{b}, \mathrm{c})$ and is used to simulate laboratory experiments undertaken by IRSN before application to more complex hydro-mechanical conditions posed by the presence of a technological void (a designed annular gap around the sample) and loss of confinement. Finally, a fullscale in situ performance test at Tournemire underground rock laboratory with a non-uniform technological void is simulated. We show that the general features of the experimental results, such as final swelling stress and water uptake, can be matched with this simple model with nine mechanical parameters but, in order to capture the full range of behaviour, more complex modelling methods incorporating elasto-plasticity may be required.

\section{Mathematical model}

In this work, we couple Richards' equation to a nonlinear elastic mechanical model incorporating a moving finite element mesh, in order to model the complex hydro-mechanical behaviour of bentonite with a limited parameter set.

\section{The hydraulic process}

The SEALEX experiments used in Task A of DECOVALEX-2015 are primarily concerned with the saturation of 
the bentonite seal from a compacted, partially saturated state. As a result, the description of fluid flow within the medium requires the consideration of more than one fluid phase. Multiphase flow in porous media is commonly described by the mass balance equations for each phase and Darcy's law of pressure driven fluid flux. A common simplification used in soil mechanics and hydro-geology for systems where the two fluids are a liquid and a gas is that of Richards' equation (Celia et al. 1990). The assumption made in Richards' equation is that the pressure change of the gas phase is negligible and therefore can be considered constant, i.e. the gas phase is infinitely mobile. This results in a direct relationship between the capillary pressure or total suction, and the degree of saturation. A pressure-based form of Richards' equation is given for the single fluid phase as derived by Thorenz (2001):

$\frac{1}{\rho_{\mathrm{w}}} \nabla \cdot\left(\rho_{\mathrm{w}} \frac{k_{\mathrm{rel}} \boldsymbol{k}}{\mu_{\mathrm{w}}}\left(\nabla \mathrm{s}-\rho_{\mathrm{w}} \boldsymbol{g}\right)\right)-Q_{\mathrm{w}}=\left(S_{\mathrm{t}} S_{\mathrm{w}}+n \frac{\partial S_{\mathrm{w}}}{\partial s}\right) \frac{\partial s}{\partial t}$

where $S_{\mathrm{w}}$ is the degree of saturation of the medium (-), $S_{\mathrm{t}}$ is the storativity of the soil $(-), n$ is the porosity of the medium $(-), s$ is the suction $(\mathrm{Pa})$ defined as the difference between the air pressure and the liquid pressure and therefore negative in unsaturated conditions, $t$ is time (s), $\rho_{\mathrm{w}}$ is the fluid density $\left(\mathrm{kg} / \mathrm{m}^{3}\right), k_{\text {rel }}$ is the relative permeability $(-), \boldsymbol{k}$ is the intrinsic permeability $\left(\mathrm{m}^{2}\right), \mu_{\mathrm{w}}$ is the dynamic viscosity of the wetting fluid $(\mathrm{Pa} \mathrm{s}), g$ is the acceleration due to gravity in vector form of $(0,0,1)$ to ensure it acts only in the $z$ axis $\left(\mathrm{m} / \mathrm{s}^{2}\right)$, and $Q_{\mathrm{w}}$ represents a source term of fluid into the system $\left(\mathrm{m}^{3} / \mathrm{s}\right)$.

This form of Richards' equation in OpenGeoSys is solved for the primary variable of fluid pressure, i.e. suction, with the degree of saturation and fluid velocity calculated as secondary variables. Two relationships are required to satisfy this equation, the constitutive relationship between the suction and degree of saturation, known as the water retention curve, and the dependence of permeability on the saturation of the sample, known as the relative permeability.

The accuracy of the coupled solution has been shown to rely heavily on the water retention curve, so it is important to choose an appropriate constitutive relationship for the material and anticipated stress conditions (Fredlund 2002; Fredlund et al. 2012). Commonly, a direct relationship between the degree of saturation and suction derived by constant volume water retention tests is fitted with water retention curve functions such as the van Genuchten relationship (van Genuchten 1980). However, these were developed for non-expansive soils and their applicability to expansive soils has been questioned due to the volumetric behaviour of expansive clays in free swelling stress conditions (Gallipoli et al. 2003). Specifically, it has been observed that variations in sample volume can lead to differences in void ratio that affect the pore dimensions and connectivity. Consequently, application of the van Genuchten formula to expansive soils requires the water retention properties to be cast in terms of degree of saturation, suction, and specific volume (Gallipoli et al. 2003).

Alternatively, the water retention curve can also be formulated in terms of degree of saturation, suction $(s)$, and stress by directly incorporating the stress into the degree of saturation-suction relationship. This forms a state surface between suction, degree of saturation, and stress (Gallipoli et al. 2003; Dueck 2004; Dueck and Börgesson 2007). The free swell water retention experimental data provide the maximum water content at zero confining stress over the full range of applied suctions and associated changes in void ratio. Under constant volume conditions, a finite volume of water can be taken up by the sample over the full range of applied suctions, but a swelling pressure develops acting on the confining apparatus. This swelling pressure, also known as the confining pressure, is the modified effective stress (" $\sigma$ ”):

$\sigma^{\prime \prime}=\left(\sigma-u_{\mathrm{a}}\right)+\beta\left(\Delta s_{\text {rel }} S_{\mathrm{w}}\right)$

where $\sigma$ is the mechanical stress, $u_{\mathrm{a}}$ is the air pressure (assumed to be zero in Richards' equation), $\beta$ is the internal strain factor (discussed in the description of the hydromechanical coupling), $\Delta s_{\text {rel }}$ is the change in relative suction (as discussed below), and $S_{\mathrm{w}}$ is the degree of saturation. The concept of Dueck suction states that the confining stress ( $p$ in Fig. 1) is equal to the suction difference at a given water content $(w)$ between the free swell water retention curve (retention curve in Fig. 1) and constant volume water retention curve (confined retention curve in Fig. 1). This is shown in Fig. 1 where the water content at points $F$ and $G$ is equal on the two water retention curves, but corresponds to different values of suction $\left(s_{\mathrm{F}}\right.$ and $\left.s_{\mathrm{G}}\right)$. This implies that the free swell water retention behaviour

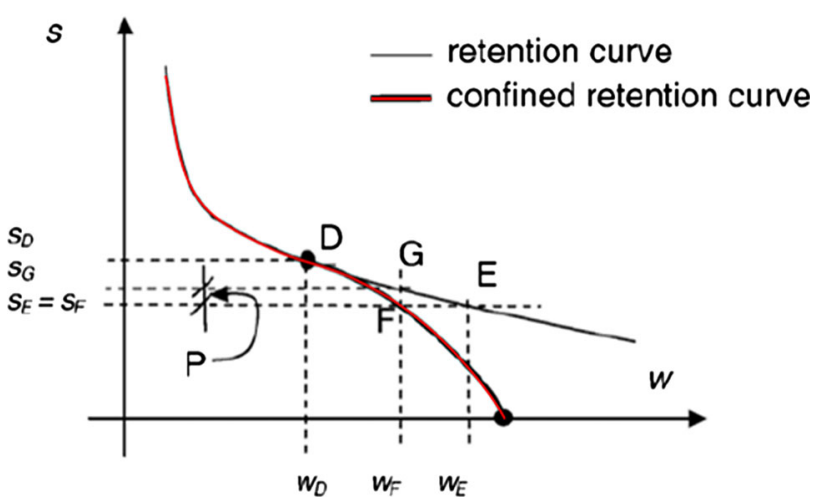

Fig. 1 A graphical representation of the Dueck suction concept (Dueck 2004; Dueck and Börgesson 2007) 
represents a limit to the maximum potential water uptake of the sample, and the constant volume water retention is a finite water uptake relative to the maximum potential. The suction that drives flow is therefore the relative suction, which is a stress-dependent parameter defined as:

$s_{\mathrm{rel}}=s_{\mathrm{fs}}-\sigma^{\prime \prime}$

where $s_{\text {rel }}$ is the relative suction (Pa) that is available to drive flow, $s_{\mathrm{fs}}$ is the free swell suction $(\mathrm{Pa})$. This allows the determination of the hydration state of the sample under a range of confining stress conditions from constant volume to free swell conditions (Dueck and Börgesson 2007). Furthermore, it provides a direct coupling between the hydraulic and mechanical processes and provides a simple method of incorporating stress-dependent water retention behaviour into the model.

Here we adopt the Dueck suction method and include a minor adaptation for consolidation stress conditions to complete the volumetric dependency of the water retention curve. Experimental data in the form of water content versus suction are converted to a degree of saturation for use in the pressure-based Richards' equation (Eq. 1). Water content by mass $(w)$ is converted to a volumetric water content $(\theta)$ defined as:

$\theta=\frac{V_{\mathrm{w}}}{V_{\mathrm{T}}}$

by multiplication with the specific gravity of the sample:

$\theta=w \mathrm{SG}$

where SG is the specific gravity of the medium defined as:

$\mathrm{SG}=\frac{\rho_{\mathrm{d}}}{\rho_{\text {ref }}}$

where $\rho_{\text {ref }}$ is the reference density of water $=1000 \mathrm{~kg} / \mathrm{m}^{3}$ and $\rho_{\mathrm{d}}$ is the dry density of the material $\left(\mathrm{kg} / \mathrm{m}^{3}\right)$. Dry density is thought to be the main control over the magnitude of swelling pressure and has therefore become a design criterion for bentonite applications as compacted blocks in a buffer or as pellets in backfill (Gens et al. 2011). Degree of saturation is calculated from:

$S_{\mathrm{w}}=\frac{\theta}{n}$

where $n$ is the porosity. It is possible to define a theoretical maximum water content a specific element can contain at saturation by substituting Eq. (6) into Eq. (7) and rearranging to give:

$w_{\max }=\frac{n}{\mathrm{SG}}$

Assuming that saturation is achieved when all voids are taken up by fluid, degree of saturation is given by:
$S_{\mathrm{w}}=\frac{w}{w_{\max }}$

The evolution of porosity during volumetric changes in the bentonite sample leads to a dependency of the degree of saturation on volume. The state surface defined by Dueck suction is applicable for stress conditions permitting constant volume and free swell behaviour (Dueck and Börgesson 2007). However, it has been noted that this approach may lead to unrealistic representations of saturation during loading-unloading experiments on clays, because irreversible void ratio changes are not taken into account by a unique water retention curve, i.e. the water content is equal for a given stress condition irrespective of whether this is achieved before or after loading (Gallipoli et al. 2003).

In this current work, the use of the free swell curve as the unique water retention curve coupled to the confining stress conditions is chosen to provide the appropriate volume-dependent water retention properties for constant volume and expansive conditions (Fig. 2, left). The retention curves shown in Fig. 1 are plotted on the state surface- the retention curve at zero confining stress (labelled, black) and the constant volume retention curve (red). In this figure, the saturation line in the stress-water content plane represents the maximum water content in the many possible degrees of confinement between constant volume conditions and free swell conditions. However, under stress conditions that lead to consolidation behaviour, i.e. external loading, the saturation line cannot remain static. In order to incorporate void ratio change in consolidation and its influence on the water retention properties, the degree of saturation limit is used to calculate the new location of the water retention curve for a given void ratio based on the theoretical local maximum water content. Consequently, the water content at a given suction is dependent on the stress state and the void ratio (or dry density), such that with decreasing void ratio the maximum water content reduces (Fig. 2). The location of the saturation line in the left-hand image of Fig. 2 is moved to a lower water content (i.e. moved towards the confining stress axis) if stress conditions leading to volume reduction prevail. This takes into account changing hydraulic properties with changing void ratio in compression and avoids a reversible water content behaviour for a loading-unloading stress path. Hysteresis of water retention properties, as experimentally shown by Ferrari and Seiphoori (2015), is not considered in this simple formulation of the stress state and volume-dependent water retention properties.

Data points are often limited in the water retention curve results at low suctions due to the large change in water content over a small range of suction. Therefore, to provide a smoothed relationship between suction pressure and 


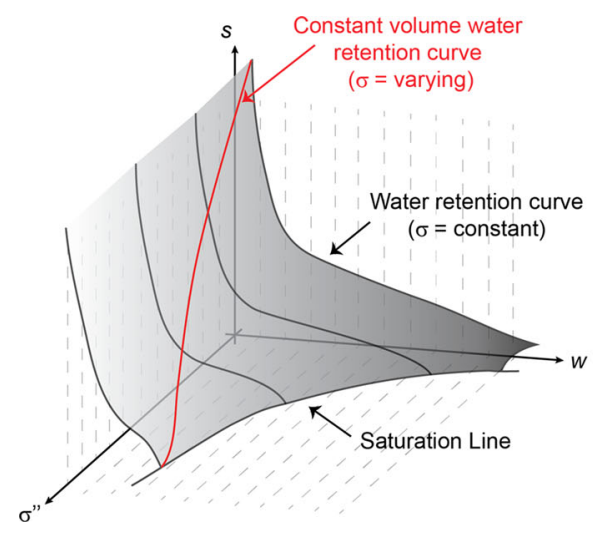

Fig. 2 Conceptual diagram of the state surface defined by the water retention properties in suction-water content-net mean stress space (left). In this case, the suction axis refers to the relative suction. The red line indicates the constant volume water retention curve as shown

water content, an empirical function proposed by (Bond and Benbow 2009) is used to match the free swell water retention data

$s_{\mathrm{fs}}=M_{\mathrm{b}}(\exp (a-b w)+\exp (c-d w))$

where $M_{\mathrm{b}}$ is the mass fraction of bentonite $(-), w$ is the water content by mass (-), and $a, b, c$, and $d$ are all fitting constants. The free swell suction given by this equation is in $\mathrm{MPa}$.

The second relationship required to fulfil Richards' equation is the relative permeability $\left(k_{\mathrm{rel}}\right)$. A simple power law is used in this work:

$k_{\mathrm{rel}}=S_{\mathrm{w}}^{\gamma}$

where $S_{\mathrm{w}}$ is the degree of saturation and $\gamma$ is the power to which the degree of saturation is raised. The power is used to calibrate the relative permeability model to the infiltration test data.

In addition to the relative permeability, in stress conditions that give rise to volumetric changes the intrinsic permeability is likely to change, especially in active clays, such as bentonite (Karnland et al. 2006). Common relationships used to determine the saturated permeability for porous media relate the permeability to porosity, e.g. the Kozeny-Carmen relationship. However, it has been shown that the $\mathrm{K}-\mathrm{C}$ relationship is most applicable to non-expansive materials and is less applicable to bentonites (Liu 2010; Liu et al. 2011). There are a variety of permeability models proposed for bentonites including the $\mathrm{K}-\mathrm{C}$ relationship, cluster model (Achari et al. 1999), gel model (Pusch and Yong 2003), macroscopic Brookes and Corey function, statistical approaches (Agus 2005), and empirical formulae (Karnland et al. 2006). Liu et al. (2011) propose an edited $\mathrm{K}-\mathrm{C}$ equation which aims to take into account the shape of the montmorillonite sheets and the montmorillonite content with respect to the solid grains and has been

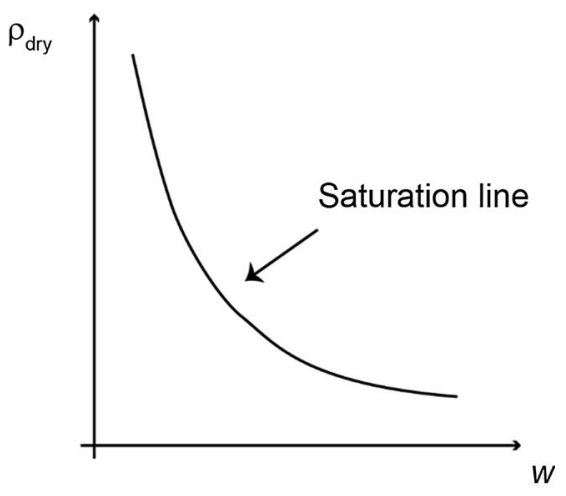

in red in Fig. 1. The right-hand figure shows that the maximum water content that a sample can contain can be described as a function of the dry density

successfully used to calculate the saturated permeability of pure MX-80 bentonite:

$k=\frac{\delta_{\mathrm{p}}^{2}}{4 C_{k} \vartheta_{\mathrm{p}}^{2}} \frac{(1-\varphi)^{m}}{\varphi^{l}}$

where $\delta_{\mathrm{p}}$ is the particle thickness, $C_{k}$ is considered as the scaled pore shape factor, $\vartheta_{\mathrm{p}}$ is the fraction of montmorillonite within the solid grains, $\varphi$ is the volume fraction of the solid, and $m$ and $l$ are calibrating model parameters, as in the Kozeny-Carmen relationship. The volume fraction of the solids is related to the porosity $(n)$ by:

$\varphi=1-n$.

This model can be used to simulate bentonites of varying montmorillonite content, including bentonite-sand mixtures, through the variation of the parameter $\vartheta_{\mathrm{p}}$ in line with the montmorillonite fraction of the material solids. This has the effect of increasing or decreasing the permeability to reflect the incorporation of accessory minerals with larger grain sizes. $\delta_{\mathrm{p}}^{2}$ and $C_{k}$ are estimated as the average values from Liu et al. (2011), and $m$ and $l$ are calibrated to fit the infiltration test. This formulation introduces the dependency of the permeability on the porosity of the sample and therefore calculates permeability for a range of dry densities. In addition, it provides a second mechanical-hydraulic coupling mechanism through the volumetric change associated with a change in intrinsic permeability.

\section{The mechanical process}

Deformations of a medium can be described in terms of stress by the momentum balance equation:

$\nabla \cdot \boldsymbol{\sigma}+\rho \boldsymbol{g}=0$

where $\boldsymbol{\sigma}$ is the Cauchy stress tensor, $\rho$ is the solid density $\left(\mathrm{kg} / \mathrm{m}^{3}\right), \boldsymbol{g}$ is a tensor with the acceleration due to gravity in 
the leading diagonal associated with the vertical direction $\left(\mathrm{m} / \mathrm{s}^{2}\right)$. In this model, the primary variable of displacement is solved for by substituting the constitutive stress-strain relationship for a linear elastic material, i.e. Hooke's Law:

$\mathrm{d} \boldsymbol{\sigma}^{\prime \prime}=\mathbf{D d} \varepsilon$

where $\boldsymbol{\sigma}^{\prime \prime}$ is here the modified effective stress tensor because suction is included in the mechanical solution as a body force, $\varepsilon$ is the strain, and $\mathbf{D}$ is the elastic constitutive matrix that defines the elastic material properties of the sample (Lewis and Schrefler 1998):

$$
\mathbf{D}=\frac{E}{(1+v)(1-2 v)}\left[\begin{array}{cccc}
1-v & v & v & 0 \\
v & 1-v & v & 0 \\
v & v & 1-v & 0 \\
0 & 0 & 0 & \frac{1-2 v}{2}
\end{array}\right]
$$

$E$ is the nonlinear Young's modulus $(\mathrm{Pa})$, and $v$ is the Poisson's ratio (-). Strain is calculated from the displacement by multiplication with the differential operator $(L)$ :

$\varepsilon=L u$.

A global system of equation is built comprising the material properties in the stiffness matrix $([K])$, the unknown displacements $(\{\boldsymbol{u}\})$, and the known body forces or surface tractions in the force vector $\{f\}$. To solve for displacements, the stiffness matrix is inverted and multiplied with the force vector:

$$
\{\boldsymbol{u}\}=[K]^{-1}\{f\}
$$

A nonlinear elastic mechanical model based on the strain history is developed to describe the mechanical behaviour of bentonite under varying stress conditions, e.g. constant volume, free swell, and consolidation. An explicit assumption for the calculation of the material properties is used, such that Hooke's Law is calculated for each timestep, but non-linearity is achieved by updating the Young's modulus as a function of the strain on the sample. The Young's modulus is updated at each time-step and is given by the following strain-dependent empirical formula:

$E_{t}=E_{t=0}\left(1+\left(\alpha \sum_{t=1}^{t-1} \Delta \varepsilon_{\mathrm{v} t}\right)^{d}\right)$

where $E_{t=0}$ is the reference initial Young's modulus $(\mathrm{Pa}), \alpha$ is the hardening or softening factor depending on the strain direction, $\varepsilon_{\mathrm{v}}$ is the total volumetric strain, and $d$ is a calibration parameter. This function leads to an increase in elastic modulus with a decrease in sample volume (i.e. strain hardening) and also a decrease in elastic modulus with an increase in sample volume (i.e. strain softening). The initial void ratio of the sample is used as a control to switch between the softening and hardening factors in the calculation of the Young's modulus. This allows softening and hardening parameters that reflect the different stress states and strain directions to be defined separately. As a result, four different conditions are considered:

- volume reduction at void ratios greater than the initial void ratio,

- volume expansion at void ratios greater than the initial void ratio,

- volume reduction for void ratios less than the initial value, and

- volume expansion for void ratios less than the initial value.

For the first two cases, the hardening and softening parameters are equal to the user-defined softening parameter. For the third case, the hardening parameter takes the user-defined value, and for the final case linear elastic rebound is assumed by calculating a constant Young's modulus.

Analysis of this empirical formula shows that calculations of large expansive strains could lead to an elastic modulus $\leq 0.0$. This is because the formula was initially developed in line with the experimental consolidation tests. Therefore, it requires extension to account for stress conditions that permit large expansive strains in order to ensure that the elastic modulus is always $>0.0$. This extension only applies in expansion and when the calculated Young's modulus is below a defined threshold. The Young's modulus used in the simulation step is then:

$E_{t}= \begin{cases}E_{t=0}\left(1+\left(\alpha \sum_{t=1}^{t-1} \Delta \varepsilon_{v t}\right)^{d}\right) & \text { for } E_{t}>E_{\min } \\ \left(E_{\min } \sum_{t=1}^{t-1} \Delta \varepsilon_{\mathrm{v} t}\right) \exp (k) & \text { for } E_{t}<E_{\min }\end{cases}$

where $E_{\min }$ is a user-defined Young's modulus threshold $(\mathrm{Pa})$, below which the effect of volumetric strain on reducing Young's modulus is reduced, and $k$ is a negative exponent that controls the rate of reduction in Young's modulus with continued volumetric strain. As water content is closely linked to expansive volumetric strains in free swell conditions, this function implies that at higher suctions a small change in water content has a larger effect on the mechanical material properties than at low suctions. This aims to reflect the transition from dry, stiff conditions towards a gel so that at low suctions and large volumetric strains further expansion occurs with a less pronounced change in mechanical material parameters.

The mechanical formulation is solved to give nodal displacements, stresses, and strains using the Galerkin finite element method in the open source code OpenGeoSys (Kolditz et al. 2016). In this current work, the 
nodal displacements are then used to update the mesh coordinates to produce a moving mesh known as an Updated Lagrangian mesh. The new element volume is used to calculate the volumetric strain, which is then used to calculate the new element void ratio, porosity, permeability, and dry density, as well as the new Young's modulus.

Void ratio $(\eta)$ is determined from the incremental volumetric strain as:

$\eta=\eta_{0}+\left(\Delta \varepsilon_{\mathrm{v}}\left(1+\eta_{0}\right)\right)$

where $\eta_{0}$ is the void ratio of the element before the mesh coordinates were updated and $\Delta \varepsilon_{\mathrm{v}}$ is the increment of volumetric strain of the element. The void ratio is used to determine the element porosity $(n)$ :

$n=\frac{\eta}{1-\eta}$

A new element dry density is given by:

$\rho_{\mathrm{d}}=\frac{M_{\mathrm{s}}^{\mathrm{e}}}{V^{\mathrm{e}}}$

where $\rho_{\mathrm{d}}$ is the dry density in $\mathrm{kg} / \mathrm{m}^{3}, M_{\mathrm{s}}^{\mathrm{e}}$ is the mass of solids of the element $(\mathrm{kg})$, and $V^{\mathrm{e}}$ is the volume of the element $\left(\mathrm{m}^{3}\right)$.

This empirical formula contains six parameters: the initial Young's modulus, the hardening and softening parameters, the calibration parameter $(d)$, the minimum Young's modulus threshold, and the exponent $k$. In order to fully describe the elastic behaviour, the Poisson's ratio is also required. Other necessary inputs, such as porosity and initial void ratio, are determined from the experimental data.

\section{Coupling}

The hydraulic and mechanical processes governing the saturation of bentonite are extremely strongly coupled; a change in hydration state contributes to a change in the effective stress calculated by the mechanical process, and the mechanical stresses exert an influence over the amount of change of hydration that can occur (Laloui and Nuth 2005; Gens et al. 2006; Nuth and Laloui 2008). As a result, this coupling must be taken into account in both hydromechanic (HM) and mechano-hydraulic (MH) directions.

The coupling of the mechanical process to the hydraulic process $(\mathrm{MH})$ is achieved in two parts: an evaluation of the suction-water content relationship as a function of the confining stress at a given void ratio and an evaluation of suction at the start of hydraulic iteration based on the confining stress conditions. Both parts of the coupling are required for the implementation of the Dueck suction concept (Eq. 3) to allow for a change in mechanical stress conditions to directly affect the hydraulic potential of the sample with an explicit calculation method.

The coupling of the hydraulic process to the mechanical process (HM) is incorporated through the calculated displacements being a function of both the hydraulic stress caused by the change in hydration state of the sample (change in suction) and the externally applied mechanical stress. Consequently, the effective stress can be expressed as:

$\sigma^{\prime}=\sigma_{\mathrm{M}}+\sigma_{\mathrm{H}}$

where the subscripts $\mathrm{M}$ and $\mathrm{H}$ refer to the mechanical and hydration stresses, respectively, and $\sigma^{\prime}$ is the effective stress. The hydration stress is a function of the suction change and is determined from the change in water content calculated by the hydraulic solution. In the mechanical solution, the suction is treated as a body force as it simultaneously acts to hold the sample together and produce a hydraulic gradient that drives fluid flow. Therefore, a change in suction leads to a change in the stress state of the sample, e.g. an increase in hydration reduces the suction pressure holding the sample together and leads to a volumetric expansion.

However, not all of suction change contributes to effective stress and subsequent deformation (Likos and Lu 2006). At low water contents, a reduction in suction causes swelling of clay clusters that is initially accommodated by crystalline swelling of aggregates into the macro-void spaces (Likos and Lu 2006; Wayllace 2008). As a result, only a small portion of the suction change contributes to effective stress. However, at higher water contents the bentonite aggregates have expanded to fill the macro-void spaces (under constant volume conditions) and the majority of further suction change contributes to effective stress, i.e. there is no space for internal deformation, and therefore, suction change translates directly to stress build-up. A similar process occurs if the sample is unconstrained, but the suction change contributes to swelling (Likos and $\mathrm{Lu}$ 2006). At low water contents, bentonite aggregates swell into macro-voids and the bulk volume change for a given suction change is less than at high water contents where large volume change occurs for a small suction change (Likos and Lu 2006). This is in part because the water adsorption mechanism transitions from crystalline swelling, which is dominant from high suction to around $20 \mathrm{MPa}$, to osmotic swelling which is dominant from around $0.8 \mathrm{MPa}$ suction (Wayllace 2008). Typically for expansive clays, it is assumed that the intra-aggregate voids are saturated, i.e. crystalline swelling is complete, before osmotic swelling predominates at water contents of $>40 \%$ (Madsen and Müller-Vonmoos 1989; Wayllace 2008).

In order to represent both the accommodation of aggregate swelling by the macro-porosity and the transition 
from crystalline to osmotic swelling processes, a factor, termed here the internal strain factor $(\beta)$, is applied to determine the contribution of hydraulic stress to effective stress. The contribution is also a function of the degree of saturation, such that hydraulic stress is given by:

$\sigma_{\mathrm{H}}=\Delta s_{\mathrm{rel}} \beta S_{\mathrm{w}}$

where $\Delta s_{\text {rel }}$ is the change in suction within the sample (a negative value in hydration), $\beta$ is the internal strain factor, and $S_{\mathrm{w}}$ is the degree of saturation.

Substituting Eq. (24) into Eq. (23) and using the notation of Bishop's effective stress, this formulation can be shown to be similar to the use of Biot's constant and gives the modified effective stress (as in Eq. 2):

$\sigma^{\prime \prime}=\left(\sigma-u_{\mathrm{a}}\right)+\beta\left(\chi s_{\text {rel }}\right)$

where $\sigma^{\prime \prime}$ is the modified effective stress, $\left(\sigma-u_{\mathrm{a}}\right)$ is the net stress, and $\chi$ is the Bishop's parameter that is here assumed to be effectively equivalent to the degree of saturation $\left(S_{\mathrm{w}}\right)$ (Lewis and Schrefler 1998).

In unsaturated non-expansive materials, Biot's constant is a proportionality factor between the suction of the fluid in the pores caused by the capillarity effect and the effective stress that is responsible for volumetric deformation (Lewis and Schrefler 1998). However, Agus et al. (2010) indicate that the dry density of bentonite, and therefore pore space size and shape, has a minimal contribution to the total suction. They conclude that suction in an expansive soil is dominated by the physico-chemical hydration forces within a clay aggregate. Following the interpretation that hydrating fluid is taken up into the clay aggregates in preference to filling the macro-porosity (Kröhn 2003), the influence of pore fluid pressure is minimal. Instead, the influence of the intra-aggregate suction is dominant and the swelling processes of aggregates define the contribution of hydraulic stress to the total effective stress.

Instead of representing an effect of pore fluid pressure, the internal strain factor therefore represents the coupled hydro-mechanical effect of the double structure of porosity observed in bentonites and the swelling mechanism operating at different water contents (Madsen and Müller-Vonmoos 1989; Lloret et al. 2003; Agus 2005; Pusch and Yong 2006; Wayllace 2008; Navarro et al. 2015). Furthermore, this process is dry density dependent because the volume of macro-void space that can accommodate expanding clay clusters is reduced for a highly compacted sample in comparison with a low dry density sample (Fig. 3) (Lloret et al. 2003; Agus 2005; Agus et al. 2010; Wang et al. 2012, 2014; Wang 2012a; Saba et al. 2014a).

Due to the non-linearity in the relationship between suction and mechanical stress, the coupling procedure is solved iteratively via a Picard iteration between the processes. The staggered, explicit iterative procedure employed is shown in Fig. 4, where $s_{\text {rel }}$ is the net suction $(\mathrm{Pa}), s_{\mathrm{fs}}$ is the free swell suction $(\mathrm{Pa}), w$ is the gravimetric water content $(\%), \sigma^{\prime \prime}$ is the modified effective stress $(\mathrm{Pa})$, $\left(\sigma-u_{\mathrm{a}}\right)$ is the external confining mechanical stress $(\mathrm{Pa})$, $E$ is the Young's modulus (Pa), $n$ is the porosity (\%), $\eta$ is the void ratio, $\rho_{\mathrm{d}}$ is the dry density of the material $\left(\mathrm{kg} / \mathrm{m}^{3}\right)$, and superscript $i$ refers to the iteration number.

The suction relative to the maximum potential suction at a given water content is used from the previous timestep (or initial conditions) as input to the hydraulic model as calculated from Dueck suction (Eq. 2). The hydraulic process calculates a new fluid pressure and water content via the saturation-dependent relative permeability function. The resulting change in hydration state is used to calculate any displacements and a new modified effective stress in the mechanical solution, taking into account any external forces $\left(\sigma-u_{\mathrm{a}}\right)$. If the system is perturbed by a mechanical stress, this leads to change in the hydraulic properties in the pre-iteration calculation of the relative suction via the Dueck suction concept, i.e. a change in mechanical conditions causes a change to the initial hydraulic conditions for the next iteration. As a result of the dependence of the hydraulic properties on the modified effective stress (Eq. 2), an iterative procedure is required between these processes such that the modified effective stress between two iterations is within a predefined tolerance:
Fig. 3 Schematic diagrams representing the difference in macro-void space in bentonitesand mixtures of different dry densities (modified after Agus et al. 2010). "M" refers to montmorillonite, "Qtz" refers to quartz, and the other particles are silt sized particles
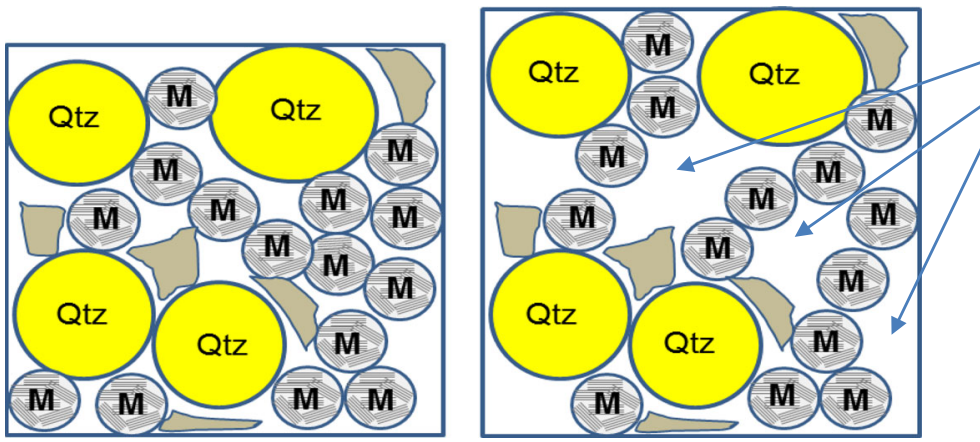

Examples of macrovoids in a low dry density sample 
$|\mathrm{err}|=\sigma^{\prime \prime(i)}-\sigma^{\prime \prime(i-1)}$

Convergence $=\mid$ err $\mid \leq$ tolerance

Calculated displacements lead to a change in the volume of a sample and a change in porosity, dry density, and permeability. Therefore, the hydraulic solution needs updating with each iteration to ensure the consistency of the hydromechanical coupling. To achieve this, we calculate the hydraulic process over an intra-time-step Updated Lagrangian mesh defined with each iteration (Fig. 4). Once the iterations have converged, the calculated displacements are used to update the nodal coordinates and the volumedependent parameters for both processes for the start of the next time-step.

In the following sections, this model is applied to a series of experiments, known as the SEALEX experiments, conducted by the Institut de Radioprotection et de Sûreté Nucléaire (IRSN) at the Tournemire underground research laboratory.

\section{SEALEX experiments and model results}

The SEALEX experimental programme comprises three main stages: a series of laboratory tests (that form modelling Steps 0 and 1), a hydro-mechanical performance test of the host rock (that forms modelling Step 2), and a fullscale in situ performance test of a bentonite core in the host rock (that forms modelling Step 3). This work is primarily concerned with the development of a hydro-mechanical bentonite model and therefore presents results for Step 0, Step 1, and Step 3.

The SEALEX experiments, as described in Barnichon and Deleruyelle (2010), Barnichon et al. (2012) and Wang (2012), investigate the hydro-mechanical behaviour of a potential hydraulic seal. A 70:30 MX-80 bentonite-sand mixture compacted to dry densities of 1.67 and $1.97 \mathrm{Mg} /$ $\mathrm{m}^{3}$ with an approximate water content of $11 \%$ by mass were used in both the laboratory tests and field performance test. MX-80 bentonite is a sodium-dominated bentonite that has a montmorillonite component of $92 \%$ in this experimental programme (Wang 2012).

The laboratory tests consist of two main stages: standard laboratory tests that make up the results for the Step 0 modelling task and a 1:10 scale mock-up test of the in situ water injection test that makes up Step 1. The Step 0 tests were designed to be used to parameterise the chosen or developed model and comprised confined and unconfined water retention tests, constant volume infiltration, and constant suction oedometer consolidation tests (Barnichon et al. 2012; Wang 2012).

For the consolidation tests, three samples were compacted to a dry density of $1.67 \mathrm{Mg} / \mathrm{m}^{3}$, emplaced in the oedometer with no annular void, and hydrated to an imposed suction of $38,12.6$, and $4.2 \mathrm{MPa}$, respectively, before consolidation was performed. The fourth consolidation test was performed on a bentonite made up of the same composition compacted to $1.97 \mathrm{Mg} / \mathrm{m}^{3}$ dry density and hydrated to a suction of $0 \mathrm{MPa}$ (Wang 2012).

For Step 1, the experimental set-up comprised a 0.12-mlong bentonite sample compacted to $1.97 \mathrm{Mg} / \mathrm{m}^{3}$ dry density with a diameter of $0.0555 \mathrm{~m}$ emplaced in a hydration cell with a diameter of $0.06 \mathrm{~m}$. This produced a symmetric technological void which was initially flooded with water. The 1:10 scale mock-up test was then divided into three main phases, with Phase 2 further subdivided into $2 \mathrm{a}$ and 2b:

- Phase 1: Initial saturation phase: flooding of the technological void and subsequent hydration from both the void and the base of the sample. During this phase, axial deformation was restrained and the build-up of axial stress was measured. The injected water volume was also measured.

- Phase 2a: The void recovery phase was initiated after axial stress equilibrium and involved the release of the vertical constraint with hydration from the base only.

- Phase 2b: Additional hydration from the top surface after $2.8 \%$ axial strain had accumulated.

- Phase 3: The confinement phase began after $20 \%$ axial strain when constant height conditions were imposed again and the stress build-up observed.

The third modelling step was the full-scale in situ performance test PT-A1. This consisted of a 1.2-m-long compacted bentonite-sand core with a diameter of $0.56 \mathrm{~m}$ emplaced in a horizontal drift hole with a diameter of $0.6 \mathrm{~m}$ leading to a non-symmetric technological void. Relative humidity was recorded at specific intervals along the core, axial stress at each end, and radial stress half way along the core at evenly spaced intervals around the circumference of the core (Barnichon et al. 2012; Wang 2012).

In the following sections, we present the results of the model from each of the three steps.

\section{Step 0}

The Step 0 modelling step consists of the water retention test, the constant volume infiltration test, and consolidation tests at imposed suctions.

\section{Water retention curve}

The water retention curve is calibrated to the free swell water retention test results using the empirical formula defined in Eq. (9). The HM coupling scheme based on the 
Fig. 4 Iterative procedure for the coupling of the hydraulic and mechanical processes

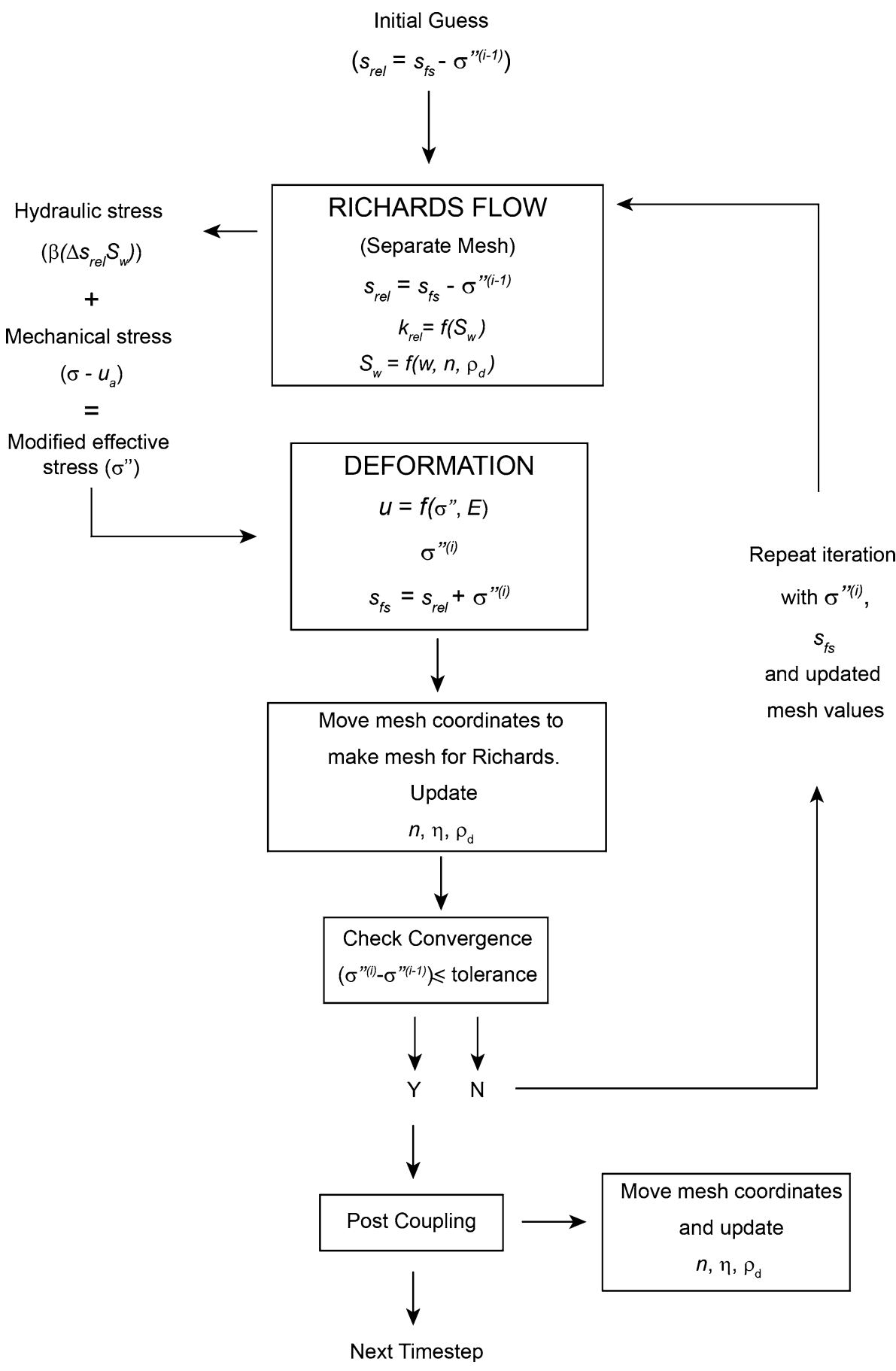

Dueck suction method leads to a confined water retention curve calculated by the model as a function of the confining stress (and void ratio during consolidation). Two experiments under free swell and constant volume conditions were conducted on pre-compacted bentonite at a dry density of $1.67 \mathrm{Mg} / \mathrm{m}^{3}$ with an initial water content of $11 \%$ and initial suction of $65 \mathrm{MPa}$ calculated from the relative humidity. Relative humidity is related to the suction $(s)$ through Kelvin's law:
$s=\frac{-R \cdot T}{M_{\mathrm{w}}\left(\frac{1}{\rho_{\mathrm{w}}}\right)} \cdot \ln R H$

where $R$ is the gas constant $(8.314 \mathrm{~J} / \mathrm{mol} / \mathrm{K}), T$ is the absolute temperature $(\mathrm{K}), M_{\mathrm{w}}$ is the molecular weight of water $(18.016 \mathrm{~kg} / \mathrm{kmol}), \rho_{\mathrm{w}}$ is the unit weight of water in $\mathrm{kg} / \mathrm{m}^{3}$, and $\mathrm{RH}$ is the relative humidity (Blatz et al. 2008). Initial relative humidity is $62 \pm 1 \%$ which corresponds to $64.5 \pm 1.5 \% \mathrm{MPa}$ suction. Figure 5 shows the calibration 
Fig. 5 Calibrated free swell water retention curve (red) matched to the experimental data for the free swell water retention test. Also shown is the calculated constant volume water retention properties from a numerical simulation (blue) compared to the experimental water retention data for constant volume conditions
Experimental Free-Swell suction curve $\Delta$ Experimental Constant volume suction curve

-Calibrated free swell suction curve

- Model calculated confined suction curve

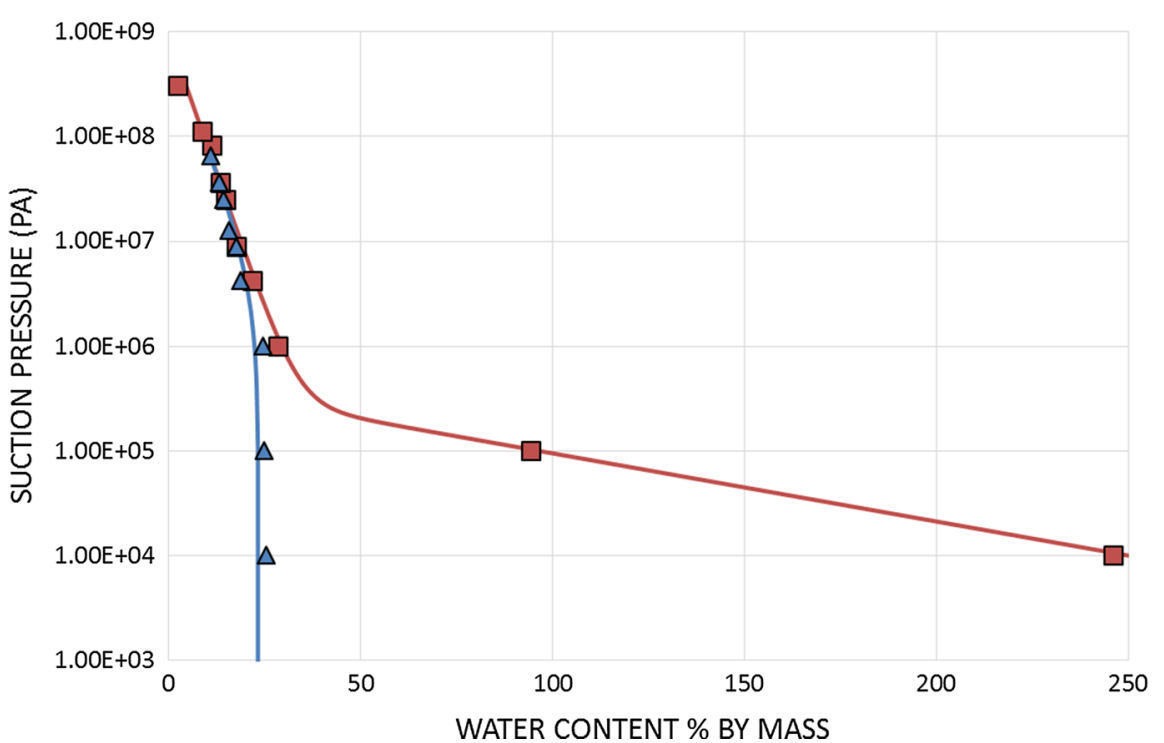

to the free swell curve (red) using the following values for the empirical constants in Eq. (10): $a=7.4, b=24.25$, $c=-0.5, d=1.5$. The mass fraction of bentonite $\left(M_{\mathrm{b}}\right)$ is 0.7 .

This calibrated curve was then used throughout the simulations with the Dueck suction concept to determine the water content at any given stress state and suction condition. Figure 5 also shows the confined water retention data calculated by the model for a constant volume simulation (blue) as a demonstration of the applicability of the approach.

\section{Infiltration test}

The infiltration test in Step 0 was performed in a metallic cylinder $250 \mathrm{~mm}$ high with a diameter of $50 \mathrm{~mm}$ and capped to maintain constant volume conditions. A precompacted bentonite sample with dry density of $1.67 \mathrm{Mg} /$ $\mathrm{m}^{3}$ and a water content of $11 \%$ was placed in the cylinder and hydrated from the base. Relative humidity was measured at four sensors through the sample: 50, 100, 150, and $200 \mathrm{~mm}$.

A 2D axisymmetric fully coupled HM model was calibrated to the experimental data. The results are shown in Fig. 6. The (Liu et al. 2011) permeability model and relative permeability are calibrated as shown in Table 1 . The coupled hydro-mechanical model is able to reproduce the general trends of the simple hydration test at the sensors from 100 to $200 \mathrm{~mm}$ from the water inlet source in the SEALEX experiments (left of Fig. 6). Final hydration states at all sensors are calculated to within $2 \%$ relative humidity. However, the rapid hydration observed at the lowest sensor (red) is under predicted. This is a common problem throughout the modelling teams in the DECOVALEX-2015 task (see summary paper this issue, Millard et al. 2016) and could be due to the permeability model not capturing the full range of permeability change associated with the hydration or the use of a single unique water retention curve to describe the water content-suctionstress relationship rather than specifically addressing the different scales of porosity.

The applicability of this model is shown in Fig. $6 \mathrm{~b}$ by $\mathrm{HM}$ modelling of the infiltration test performed on the Gao-Miao-Zi (GMZ) bentonite by Ye et al. (2009). The GMZ bentonite is compacted to a different dry density, is a pure bentonite (i.e. $M_{\mathrm{b}}=1.0$ ), and has a significantly different chemical make-up [less $\mathrm{Na}^{+}$and more $\mathrm{Ca}^{2+}$ and $\mathrm{Mg}^{2+}$ (Ye et al. 2009)]. Nevertheless, the permeability model is able to give a good fit to the experimental data with only a very minor change to the parameterisation to account for all these differences (Table 1). The results indicate that this model can be applied to model bentonite and provides a good calibration to the SEALEX Step 0 data as a good starting point for the later modelling steps.

\section{Consolidation tests}

The consolidation tests in Step 0 were performed at different imposed suctions of $0,4.2,12.6$, and $38 \mathrm{MPa}$. The $0 \mathrm{MPa}$ suction case was performed on a bentonite compacted to a dry density of $1.97 \mathrm{Mg} / \mathrm{m}^{3}$ and trimmed to diameter of $35.13 \mathrm{~mm}$ to leave a technological void around the edge of the sample. The higher suction tests were conducted on samples prepared in the same manner as the 

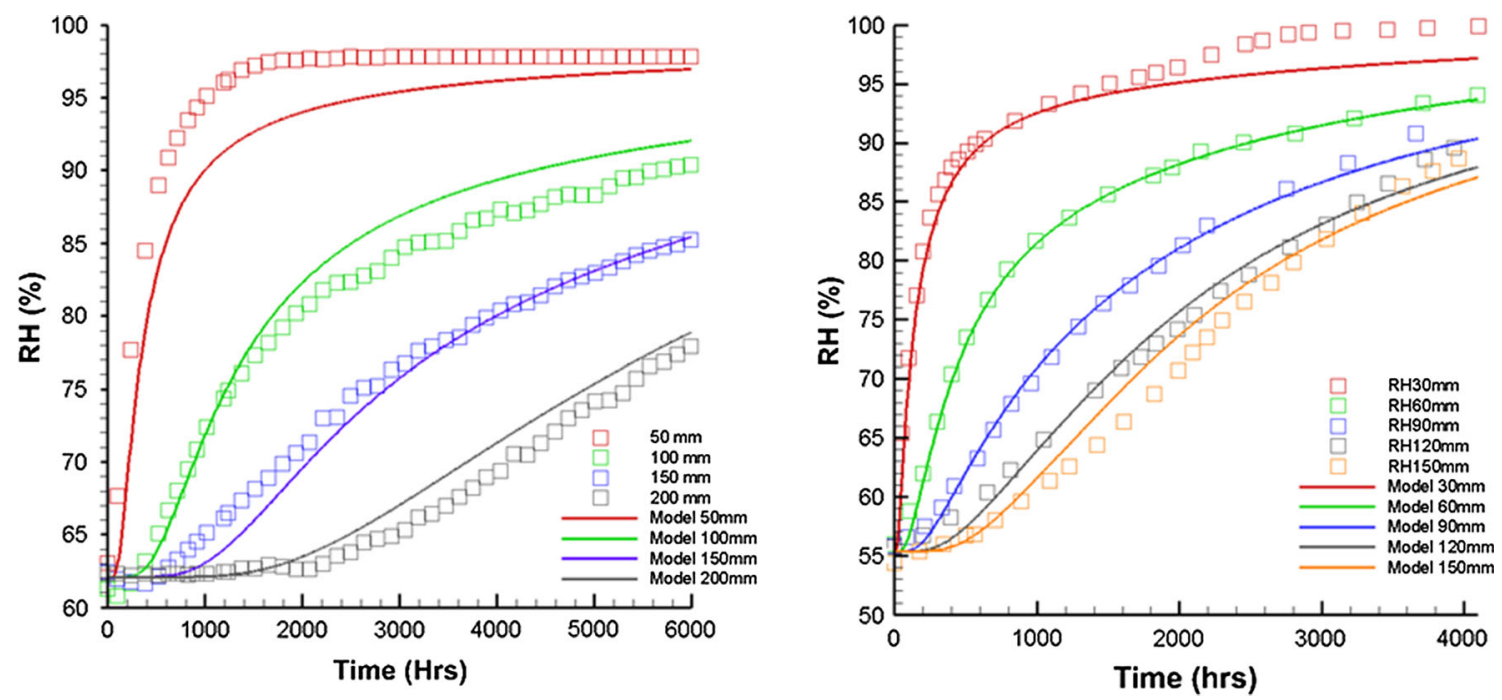

Fig. 6 Calibrated results for the infiltration tests for two bentonites using a 2D axisymmetric fully coupled HM model: SEALEX left, GMZ right

Table 1 Hydraulic parameters used in the fully coupled HM model for the infiltration test on MX-80 bentonite in the SEALEX experiments and the Gao-Miao-Zi (GMZ) bentonite in Ye et al. (2009)

\begin{tabular}{lllllll}
\hline & Parameter & Value (MX-80) & Value (GMZ) & Unit & Description & Origin \\
\hline Intrinsic permeability & $\delta_{\mathrm{p}}$ & 1.2 & 1.2 & $\mathrm{~nm}$ & Ave. montmorillonite thickness & Liu et al. (2011) \\
& $C_{k}$ & 5 & 5 & - & Pore shape factor & Liu et al. (2011) \\
& $\vartheta_{\mathrm{p}}$ & $M_{\mathrm{b}} \times 0.92$ & $M_{\mathrm{b}} \times 0.75$ & - & Bentonite fraction in solids & Experimental data \\
& $m$ & 5.5 & 5.3 & - & Fitting parameter (Eq. 10) & Calibrated \\
Relative permeability & $l$ & 4 & 4 & - & Fitting parameter (Eq. 10) & Calibrated \\
\hline
\end{tabular}

infiltration test and had a diameter of $38 \mathrm{~mm}$ equal to that of the oedometer ring. There was no technological void for these samples. All the samples were hydrated from the base (and the technological void in the $0 \mathrm{MPa}$ suction case) and allowed to swell until the imposed suction. On stabilisation of the axial strain, the samples were subjected to an axial loading-unloading cycle with a maximum net axial stress of $49 \mathrm{MPa}$.

A 2D axisymmetric fully coupled HM model using the hydraulic parameters above was calibrated to the three consolidation tests conducted on the $1.67 \mathrm{Mg} / \mathrm{m}^{3}$ dry density samples. The models were run from a consistent starting material and follow the experimental procedure of swelling to an imposed suction, followed by consolidation and then unloading. The calibration presented is consistent with the mechanical model used for the later modelling steps (Step 1 and Step 3) and as such does not necessarily represent the best possible fit to the individual consolidation test results. The internal strain factor for the $1.67 \mathrm{Mg} / \mathrm{m}^{3}$ dry density samples is given in (Fig. 7), and the full mechanical calibration is given in Table 2.

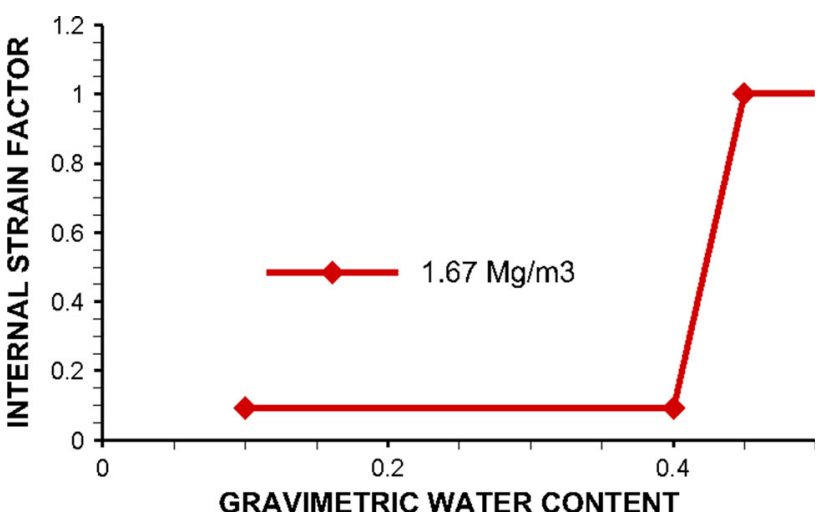

Fig. 7 Internal strain factor $(\beta)$ for $1.67 \mathrm{Mg} / \mathrm{m}^{3}$ dry density samples

The internal strain factor is low for low water contents where crystalline swelling is dominant and macro-pores accommodate volume expansion of aggregates (Likos and Lu 2006; Wayllace 2008). Osmotic swelling is thought to predominate at water contents $>40 \%$ once crystalline swelling is complete (Madsen and Müller-Vonmoos 1989; Wayllace 2008) and as such macro-pores are filled and 
Table 2 Mechanical parameters used in the fully coupled HM models for the consolidation tests

\begin{tabular}{llllll}
\hline & Parameter & Value & Unit & Description & Origin \\
\hline Elastic stiffness & $v$ & 0.30 & - & Poisson's ratio & Calibrated \\
& $E_{0}$ & 30.0 & MPa & Initial Young's modulus & Calibrated \\
& $\alpha_{\mathrm{h}}$ & 14.0 & - & Hardening parameter & Calibrated \\
& $\alpha_{\mathrm{s}}$ & 3.50 & - & Softening parameter & Calibrated \\
& $d$ & 3.0 & - & Dimensionality parameter & Calibrated \\
& $E_{\min }$ & 2.0 & MPa & Minimum $E$ threshold & Calibrated \\
$k$ & -1.0 & - & E reduction parameter & Calibrated \\
& $\beta$ & $f(w)$ & - & Internal strain factor & Calibrated \\
\hline
\end{tabular}

by this nonlinear elastic model are considered sufficient to move forward to the application of the model to more complicated hydro-mechanical conditions.

\section{Step 1}

The laboratory experiment used for the Step 1 modelling task was a 1:10 scale mock-up of the SEALEX in situ test. The experimental set-up is shown in Fig. 9 and comprises a $55 \mathrm{~mm}$ diameter by $120 \mathrm{~mm}$ height compacted bentonitesand mixture sample (with a ratio of 70:30) at a dry density of $1.97 \mathrm{Mg} / \mathrm{m}^{3}$. This was placed in a rigid hydration cell with a diameter of $60 \mathrm{~mm}$ to simulate a technological void around the bentonite sample. The technological void is symmetric in this mock-up test which differs slightly to the full-scale in situ test. The top of the cell is a piston $60 \mathrm{~mm}$ in diameter and $150 \mathrm{~mm}$ long that enables control over the axial swelling.

\section{suctions of 4.2, 12.6, and $38 \mathrm{MPa}$}

Fig. 8 Consolidation test results for the low dry density samples at

suction change is transferred directly into stress. This function is similar to the pseudo-Biot's coefficient used in Nguyen et al. (2005).

The nonlinear elastic approach is capable of reproducing the irrecoverable strains observed in the oedometer tests (Fig. 8). The model is able to adequately reproduce the behaviour of the lower suction samples, but the model calibration is unable to recreate the increasing stiffness of the higher suction sample ( $38 \mathrm{MPa}$ ). The experimental data indicate that the pre-consolidation pressure increases with increasing suction in a linear fashion (Wang 2012) and is thought to reflect the movement of the failure curve with respect to suction in elasto-plastic models, i.e. suction hardening (Gens and Alonso 1992). The nonlinear elastic model is not able to recreate this with a calibration consistent with the later modelling steps. However, this model requires just 8 parameters to be able to account for irreversible strains and reproduce the major trends of the data. As the aim of this study is to investigate an alternative model of bentonite with an emphasis on keeping the number of parameters to a minimum, the results obtained

\section{Model set-up}

A challenge of this modelling stage was how to represent the presence and effect of the technological void in the simulation. Here we use the moving finite element mesh to keep track of the bentonite swelling into the void space and when the void has been filled by the expanding bentonite. Displacement-dependent boundary conditions for both the hydraulic and mechanical processes are implemented. Once the maximum displacement of a boundary node has been reached, the hydraulic boundary condition of the technological void representing liquid hydration is removed and a zero displacement mechanical boundary introduced. These boundary conditions can be expressed as:

$$
\begin{aligned}
\mathrm{BC}_{\mathrm{H}} & = \begin{cases}s, & u<u_{\max } \\
Q=0, & u \geq u_{\max }\end{cases} \\
\mathrm{BC}_{\mathrm{M}} & = \begin{cases}\sigma_{\mathrm{c}}=0, & u<u_{\max } \\
u=0, & u \geq u_{\max }\end{cases}
\end{aligned}
$$

where $\mathrm{BC}_{\mathrm{H}}$ and $\mathrm{BC}_{\mathrm{M}}$ are the hydraulic and mechanical boundary conditions, respectively, $s$ is the suction $(\mathrm{Pa})$, 

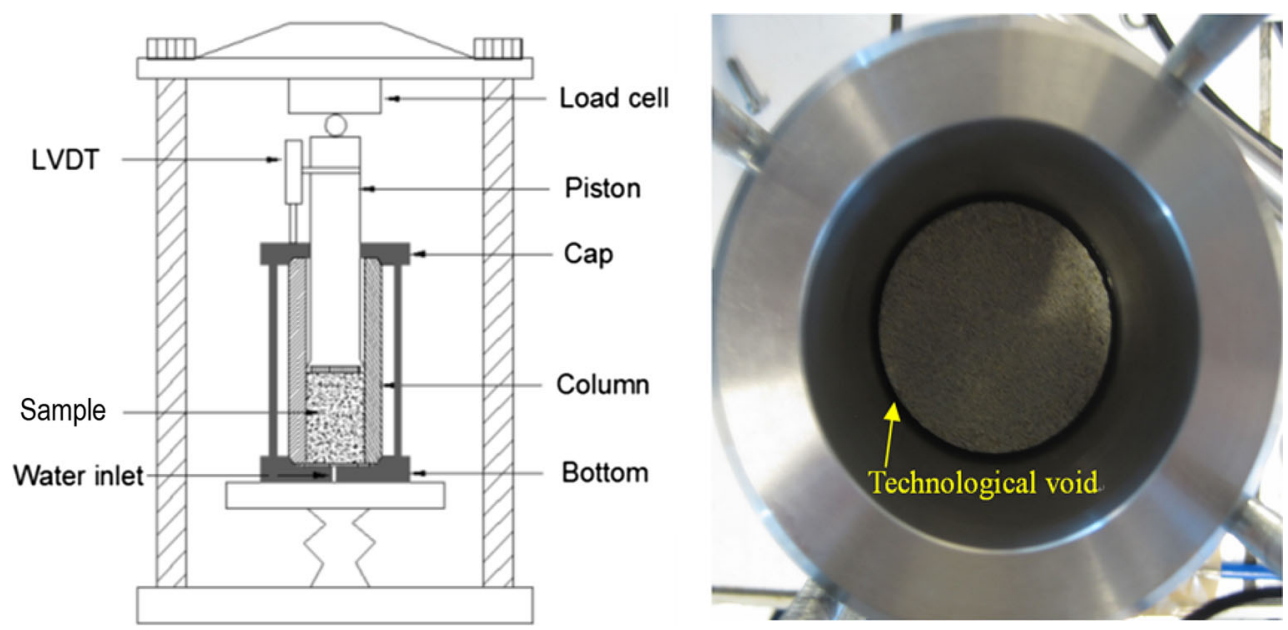

Fig. 9 Experimental set-up for Step 1 with the hydration cell schematic (left) and the pre-experiment set-up with the uniform technological void (right)

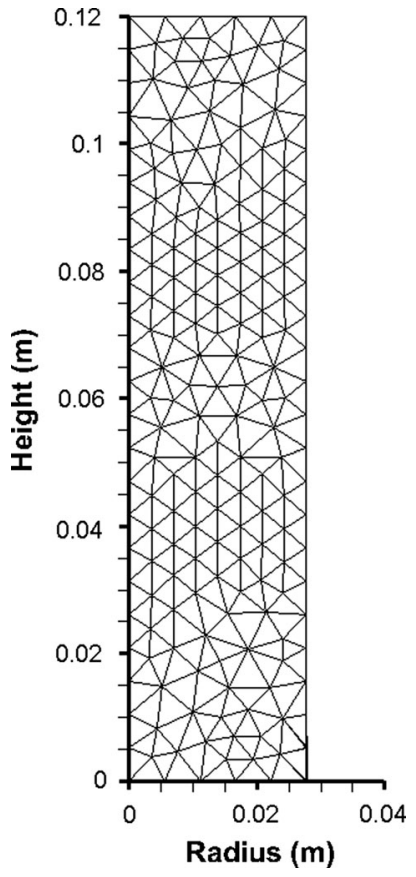

Fig. 10 2D Axisymmetric mesh used in Step 1

$Q$ is the flux $\left(\mathrm{m}^{3} / \mathrm{s}\right), \sigma_{\mathrm{c}}$ is the confining stress $(\mathrm{Pa}), u$ is the calculated displacement $(\mathrm{m})$, and $u_{\max }$ is the displacement limit (m) that represents the technological void in Phase 1 and the axial displacement in Phase 2b. This representation of the technological void defines the void to be filled with liquid water until the bentonite expansion fills the void space. It therefore does not take into account the formation of colloidal suspensions or gel that has been observed in experiment (Saba et al. 2014b).

A 2D axisymmetric mesh, shown in Fig. 10, is used with the fully coupled HM model and the Step 0 calibration presented earlier. However, the Step 1 experiments are

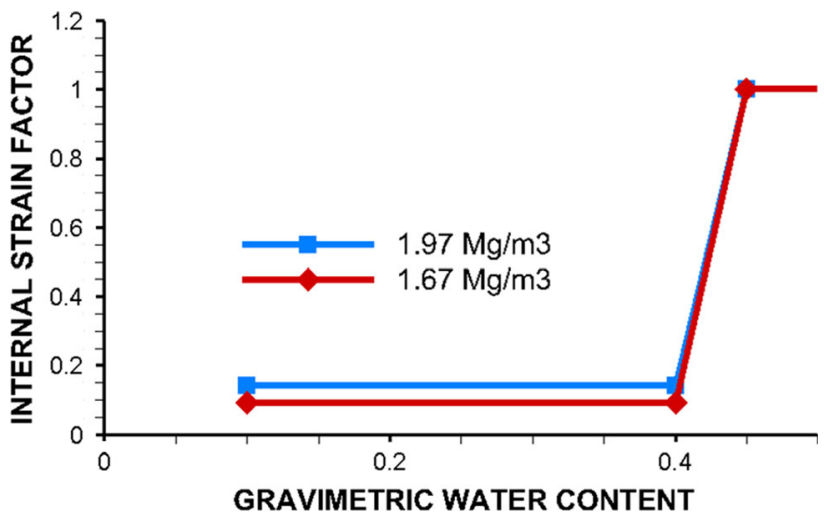

Fig. 11 Comparison of internal strain factor used for the different dry density materials

conducted on samples with a higher dry density than the calibrated model. The difference in dry density is reflected in the model through the internal strain factor. The higher dry density material has less macro-voids, and as a result clay aggregates have less space to swell into as hydration proceeds (Likos and Lu 2006). This results in a greater transfer of suction to effective stress and requires an increase in the internal strain factor (Fig. 11). This function coupled with the Dueck suction concept has the effect of ensuring that a higher dry density sample takes up less water than a lower dry density sample under constant volume conditions for a given change in suction and a higher swelling pressure for a given water content.

The Step 1 axial stress results for Phase 1 exhibit a wetting-induced collapse phenomenon that causes a reduction in axial stress as hydration proceeds. This has been observed in other experimental programmes (Lloret et al. 2003; Romero et al. 2003; Gens et al. 2011) during hydration under constant volume conditions, although 
more commonly in $\mathrm{Ca}^{2+}$ bentonites such as Calcigel (Lloret et al. 2003). The process has been hypothesised in a number of ways regarding the collapse of the macrostructure, e.g. as a result of cementing bonds between the grains in initially dry samples being broken by the hydrating water (Agus 2005); by collapse of the macrostructure in response to the developed swelling pressure/confining load (Lloret et al. 2003); or by structural rearrangement of clay clusters within the sample as a result of the frictional coefficient of the soil being overcome by wetting-induced shear forces at the micro-scale (Agus 2005). These mechanisms all consider the wetting-induced plastic collapse phenomenon to be as a result of the interaction between micro-structural processes and the collapse of the macro-structure.

Under experimental constant volume conditions, the collapse or rearrangement of the macro-structure leads to a reduction in axial stress, and in order to maintain the experimental constant volume conditions the applied load is reduced (Lloret et al. 2003). Collapse of the macrostructure is associated with macro-structure compressive strains which are partially offset by expansion of the microstructure to fill the voids (Lloret et al. 2003; Gatabin et al. 2016). As a result, the stress reduces until the microstructure strain leads to a further build-up in stress. This is interpreted as a plastic phenomenon, and therefore, due to the elastic assumption in this work, the simple formulation presented is not able to predict this behaviour. In order to represent these internal compressive strains and reproduce the expansive collapse phenomenon, a source term model to account for the reduction in axial stress is proposed.

The source term is a mechanical stress applied to the model and is activated by a failure curve that can be derived from experimental results. Wang (2012) identified

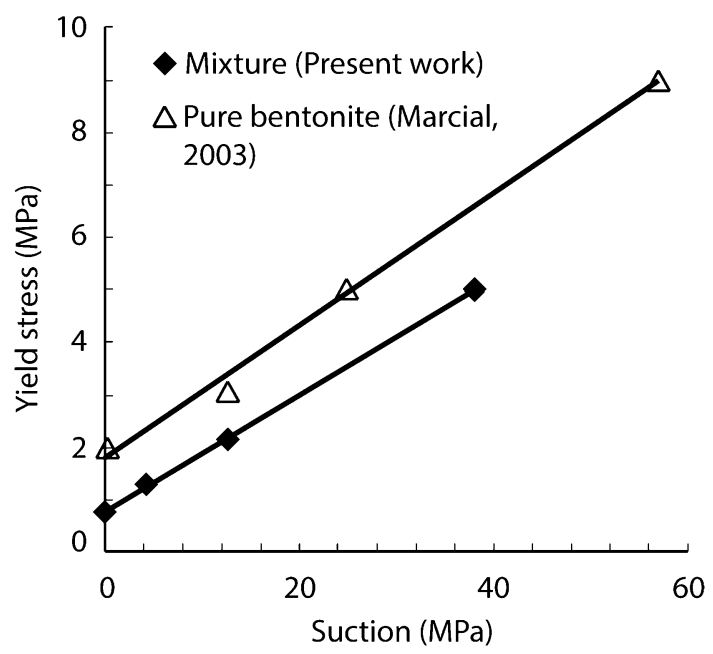

Fig. 12 A linear relationship between suction and pre-consolidation pressure form the consolidation tests in the SEALEX laboratory tests (Wang 2012) a linear relationship between the yield stress and net suction in the consolidation tests (Fig. 12). Considering the yield stress to be the axial stress at which plastic behaviour begins for the imposed net suction, the linear trend is used to define an axial stress-suction relationship that determines the activation of the source term. This is similar to the loading-collapse curve in elasto-plastic models that represents the change in yield curve location with respect to net mean stress and suction (Alonso et al. 1990; Gens and Alonso 1992; Alonso and Vaunat 1999). The calculated source term acting in the opposing direction to the build-up of axial stress is then a function of the proportion of the sample with stress-suction conditions in excess of the yield curve (Fig. 13). The dependency of the onset of the source term on the axial stress and suction provides a method to calculate the gradual development of the expansive collapse phenomenon seen in experimental results.

\section{Results of Step 1}

Experimental axial stress measurements were taken from the 60-mm-diameter piston at the top of the sample (Wang 2012) and as such represent the mean axial stress across the top surface. The model results are therefore calculated from the top surface of the 2D axisymmetric model and presented along with water uptake in Fig. 14. Model results are presented for both the nonlinear elastic model (NLE) without accounting for wetting-induced collapse and the nonlinear elastic source term model (NLE-ST).

The axial stress results for the two models (NLE and NLE-ST) show that the nonlinear elastic model over-predicts the axial stress build-up during Phase 1 as the plastic collapse behaviour is not captured. With the source term addition to the model, the final stress can be well matched and the magnitude of energy lost in the process is quantified by the gap between the NLE and NLE-ST model results. The NLE-ST model provides a good fit to the experimental data, capturing a gradual collapse process and the subsequent build-up in stress.

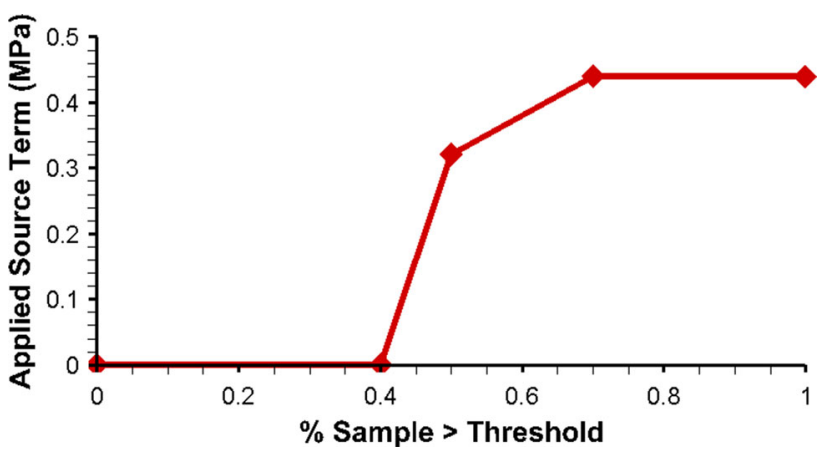

Fig. 13 Application of the source term as a function of the proportion of the sample exceeding the axial stress-net suction threshold 

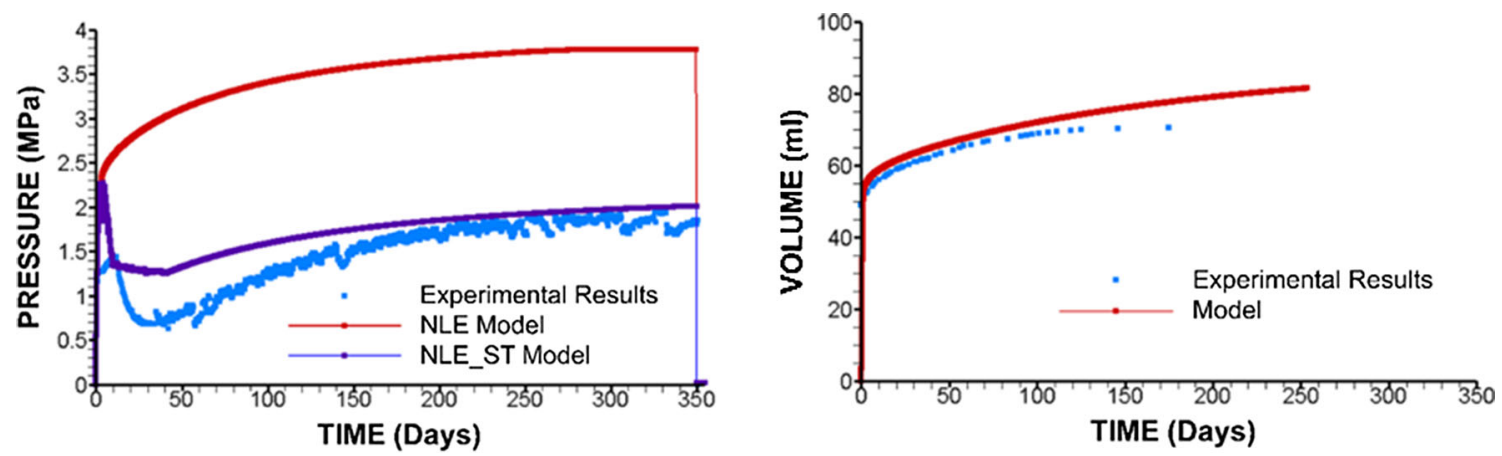

Fig. 14 Axial stress (left) and volume of water uptake (right) results for Step 1 Phase 1

The water uptake behaviour is well matched during the early stages of the model, but the maximum water is overpredicted by the end of Phase 1 . Due to the application of the boundary conditions as a mechanism to represent the technological void, the water uptake and swelling of the bentonite are calculated to occur over a short time period. The technological void is predicted to be closed after $32 \mathrm{~h}$. This rate of expansion is slower than that observed during experiments (Saba et al. 2014b) on a 35-mm diameter $1.97 \mathrm{Mg} / \mathrm{m}^{3}$ dry density sample with a $14 \%$ technological void, where radial stress builds up after less than $1 \mathrm{~h}$. It does, however, indicate that the technological void is filled quickly before the process of homogenisation proceeds due to continued swelling as the dry centre is gradually hydrated (Saba et al. 2014b).

During Phase 2, displacement develops at two distinct rates relating to the hydration conditions: slower deformation occurs initially as hydration is from the base only (Phase 2a), but more rapid deformation proceeds when hydration is from both the base and the top (Phase 2b). Displacement was measured throughout, and the model calculation from the NLE-ST model is presented in Fig. 15. The end of Phase 2a was temporally defined in the model, but the end of Phase $2 b /$ start of Phase 3 is a

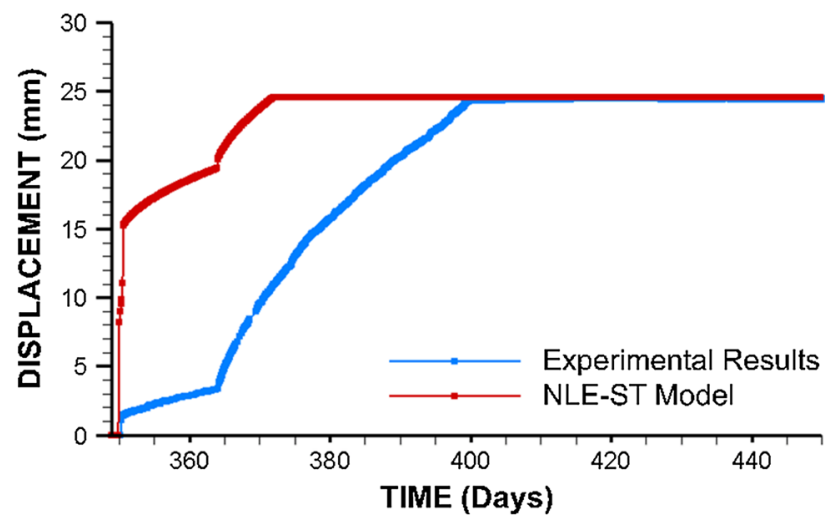

Fig. 15 Vertical displacement during Phase 2 calculated with the NLE-ST model displacement-dependent boundary condition that becomes active after $20 \%$ axial strain and is therefore a predicted result.

The elastic rebound calculated by this model is overpredicted due to the excess of stress in the system and the strain-softened material properties associated with $c .14 \%$ swelling into the technological void. The source term model does not remove any of the stress from the system that can be released from the unloading of the piston, and as a result the initial elastic rebound is too large. The activation of the source term as a function of axial stress and suction leads to the removal of the source term during Phase 2a. The release of this energy leads to a large elastic rebound which also causes the $20 \%$ axial strain to be reached twice as quickly as the experimental results.

The stress build-up during Phase 3 is shown in Fig. 16.

The model predicts an excellent fit to the final stress build-up during Phase 3. Furthermore, the evolution of the stress build-up is very similar to that of the experimental results. However, due to the over-predicted rebound in Phase 2 the onset of stress build-up is too early. Comparing the stress build-up without considering the temporal prediction of Phase 2 shows an excellent match to the experimental data (Fig. 17). The main control over the development of this axial stress in Phase 3 is the suction

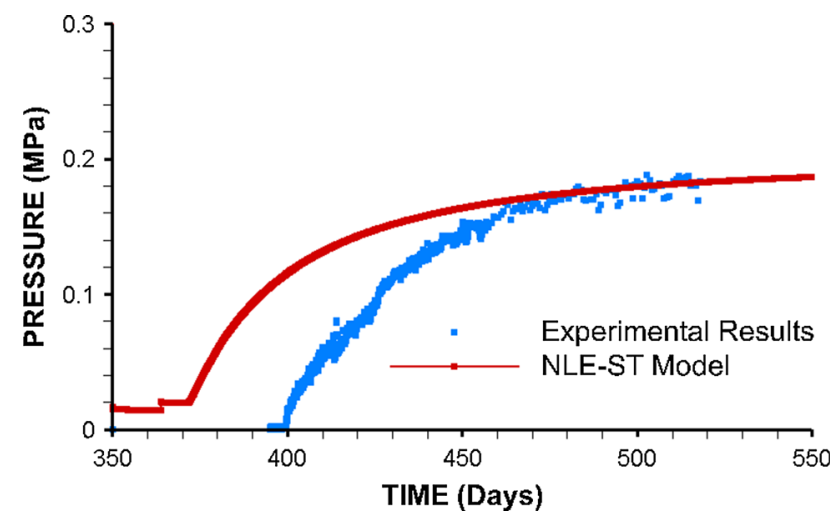

Fig. 16 Axial stress build-up during Step 1 Phase 3 


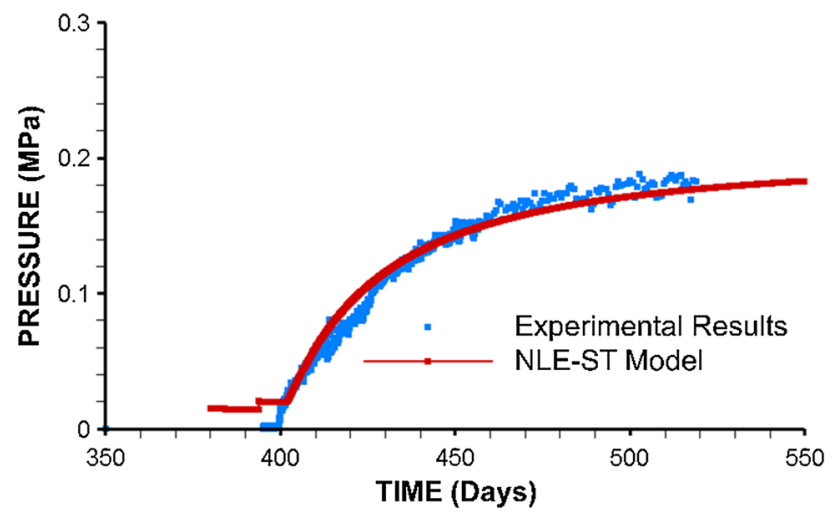

Fig. 17 Temporally adjusted axial stress build-up during Phase 3 of Step 1

remaining within the sample that can be alleviated under constant volume conditions.

Although the NLE-ST model is not able to reproduce all the experimental data, it is able to represent all the major steps of the complex hydro-mechanical behaviour with just six mechanical parameters and an experimentally defined stress threshold. Final axial stress calculations and water uptake calculations match well with experimental data, but the temporal evolution of the deformation is found harder to predict without the consideration of plasticity.

\section{Step 3}

The third modelling step of the SEALEX experiments is of the full-scale in situ test PT-A1 (Barnichon et al. 2012). The PT-A1 performance test consisted of a bentonite-sand core $1.2 \mathrm{~m}$ in length and $0.56 \mathrm{~m}$ in diameter emplaced in a horizontal drift hole with a diameter of $0.60 \mathrm{~m}$. Constant volume conditions were maintained by a confining plug in the gallery end and water inlets at both ends connected to the same water tank. Due to the horizontal emplacement, the technological void was non-uniform around the sample as shown in Fig. 18. Water was injected into the system under $0.2 \mathrm{MPa}$ pressure to ensure the technological void was flooded. Hydration proceeded from the technological void and through the sample ends. The bentonite-sand core contained relative humidity sensors at 0.22 (RH22-1, RH22-2) and $0.52 \mathrm{~m}$ (RH52-1, RH52-2), axial stress sensors at both ends, and radial stress sensors evenly spaced around the edge of the sample at $0.6 \mathrm{~m}$ (Fig. 18).

\section{Model set-up}

The non-uniform nature of the technological void presents a challenge for a 2D axisymmetric approach so this is supplemented with a 2D slice model at $0.60 \mathrm{~m}$. The Step 3 $2 \mathrm{D}$ axisymmetric model is an upscaled version of the Step
1 mock-up test. The respective finite element meshes are presented in Fig. 19.

The displacement-dependent boundary conditions used for Step 1 are again employed in Step 3 and extended to the $2 \mathrm{D}$ slice model by defining the displacement limit from the equation for a circle with the same radius as the excavated drift hole. The boundary conditions for the 2D slice model can be expressed as:

$\mathrm{BC}_{\mathrm{H}}= \begin{cases}s, & x_{n}<x_{\max }=\sqrt{r^{2}-\left(y_{n}-r\right)^{2}} \\ Q=0, & x_{n} \geq x_{\max }=\sqrt{r^{2}-\left(y_{n}-r\right)^{2}}\end{cases}$
$\mathrm{BC}_{\mathrm{M}}= \begin{cases}\sigma=0, & x_{n}<x_{\max }=\sqrt{r^{2}-\left(y_{n}-r\right)^{2}} \\ u=0, & x_{n} \geq x_{\max }=\sqrt{r^{2}-\left(y_{n}-r\right)^{2}}\end{cases}$

where $x_{\max }$ is the calculated coordinate on the circumference of a circle with radius $r$ for the nodal coordinate $y_{n}$. This value is compared to the mesh $x$ coordinate $\left(x_{n}\right)$.

The mechanical model employed is the nonlinear elastic model without the source term approximation of plasticity. The hydraulic model is consistent with the previous steps except for an increase in permeability as shown in Table 3.

\section{Results of modelling the PT-A1 performance test}

The axisymmetric model is used to calculate the axial stress at each end of the sample: 1.2 and $0.0 \mathrm{~m}$ and the relative humidity at sensors $\mathrm{RH} 22-2$ and for both sensors at RH52. An axisymmetric model is not capable of predicting the relative humidity of $\mathrm{RH} 22-1$ due to the non-uniform technological void in the experiment so the $2 \mathrm{D}$ slice is used to investigate the potential difference in relative humidity due to the non-symmetric technological void.

The axial stress results in Fig. 20 indicate that the model provides a good fit to the experimental data for the first 180 days. After this, the experimental results plateau, but the model calculates a continued increase. The experimental results do not show the development of stress until 20 days due to the lag in hydration during the experimental procedure (Wang 2012). The gradient of the stress development matches sensor AS_000, but does not predict the non-symmetry stress development between the two sample ends. However, the physical reason for the dissymmetry in axial stress is not known (Millard et al. 2016). For example, it could be an experimental difference in stress conditions at each end of the sample, such as an unaccounted for void into which the bentonite was able to expand, rather than a physical process occurring within the sample during hydration.

The model results for relative humidity at sensors RH52-2, RH22-1, and RH22-2 are presented in Fig. 21. Relative humidity is calculated to within $1 \% \mathrm{RH}$ at RH52-2 

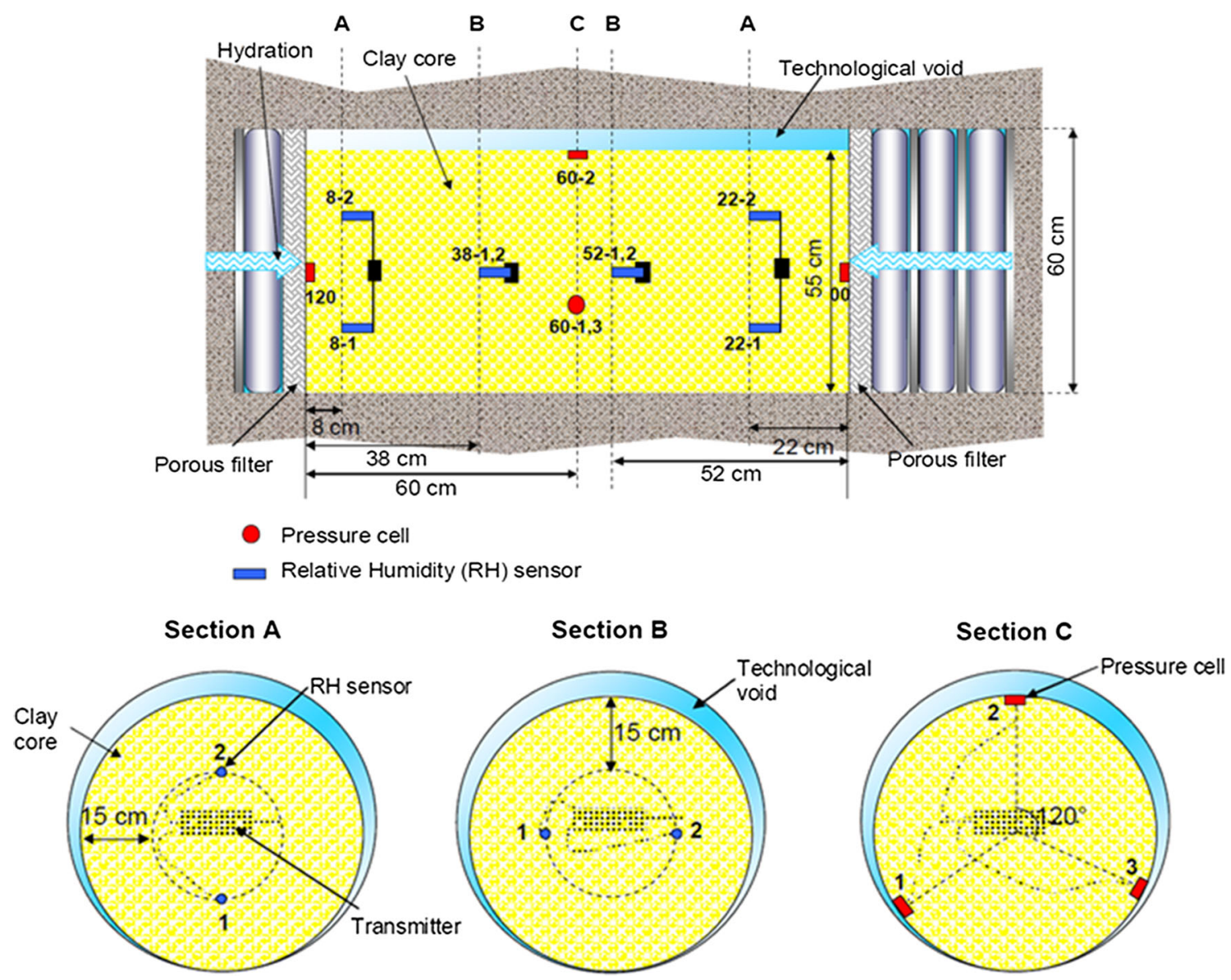

Fig. 18 Schematic of the experimental set-up for the PT-A1 performance test. The core contained multiple sensors whose location is shown in the schematic diagrams on the right. The non-uniform technological void is shown as a result of the horizontal experimental set-up
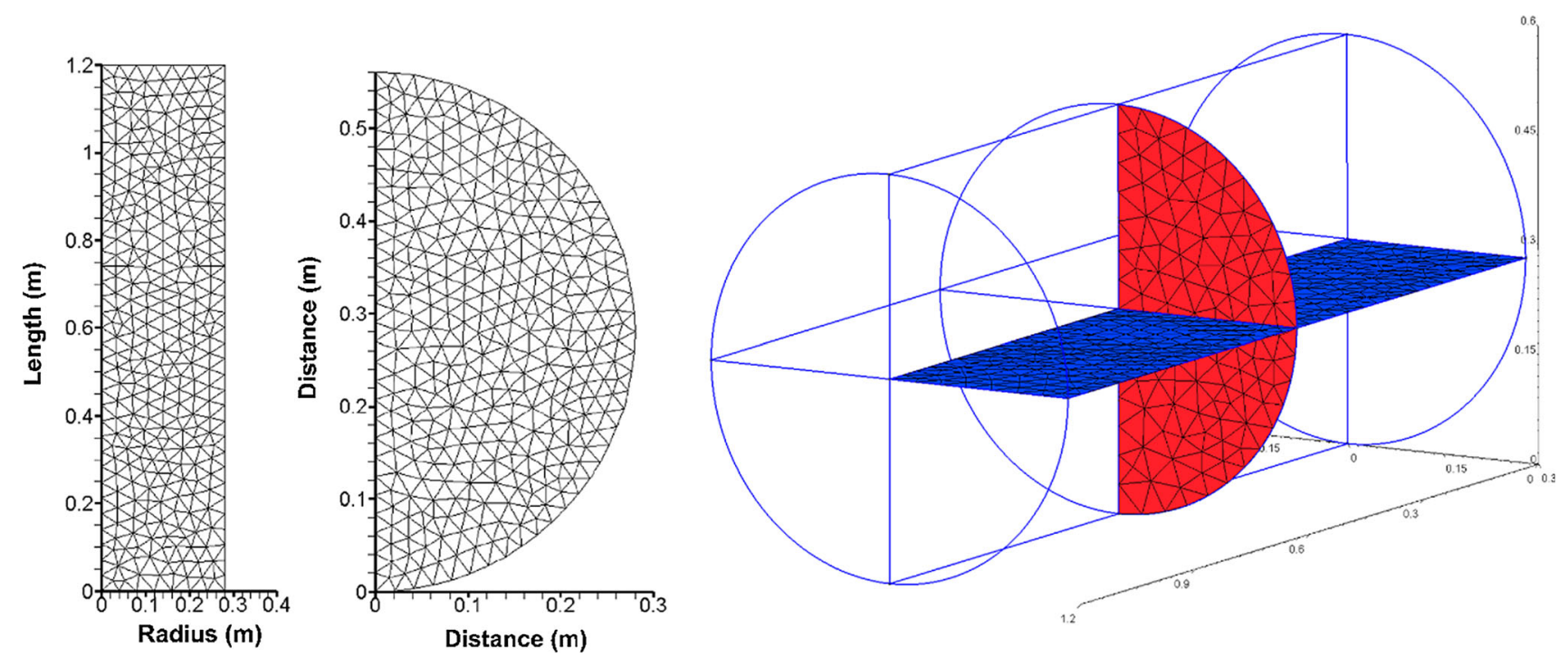

Fig. 19 2D Axisymmetric finite element mesh (left) and 2D slice mesh (centre) shown in their respective locations in the modelled 3D core (right)

with the elevated permeability model. The final magnitude of relative humidity is also closely matched at sensor RH52-2 with the imposed displacement-dependent boundary conditions suggesting a lack of fluid input once the technological void is filled. The $2 \mathrm{D}$ slice model is able to reproduce the difference in relative humidity between 
Table 3 Hydraulic parameters used to model PT-A1 experiment

\begin{tabular}{llllll}
\hline & Parameter & Value & Unit & Description & Origin \\
\hline Intrinsic permeability & $\delta_{\mathrm{p}}$ & 1.2 & $\mathrm{~nm}$ & Ave. montmorillonite thickness & Liu et al. (2011) \\
& $C_{k}$ & 5 & - & Pore shape factor & Liu et al. (2011) \\
& $\vartheta_{\mathrm{p}}$ & $M_{\mathrm{b}} \times 0.92$ & - & Bentonite fraction in solids & Experimental data \\
& $m$ & 5 & - & Fitting parameter (Eq. 10) & Calibrated \\
Relative permeability & $l$ & 4 & - & Fitting parameter (Eq. 10) & Calibrated \\
\hline
\end{tabular}

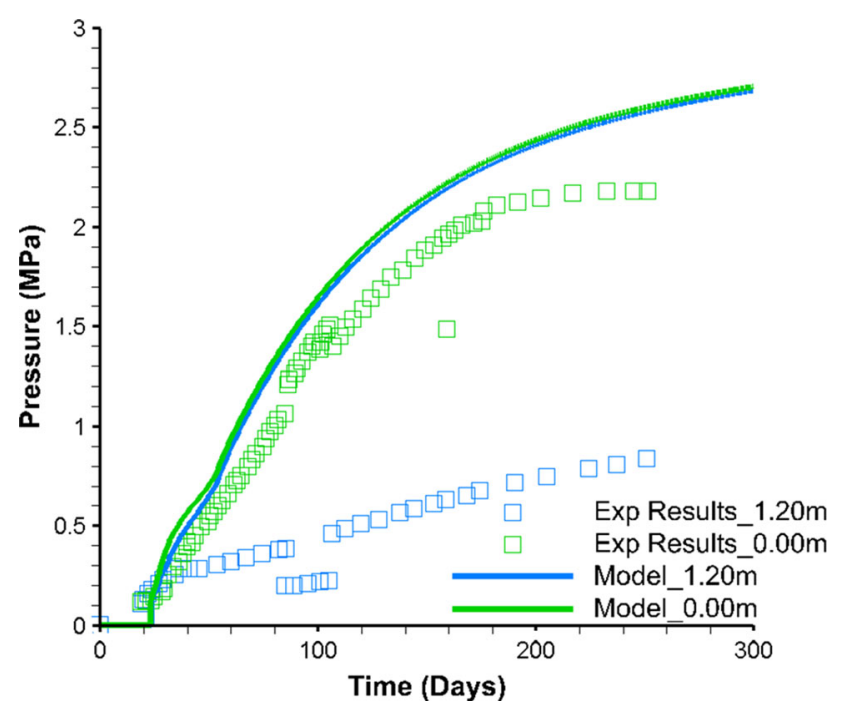

Fig. 20 Axial stress calculated by the nonlinear elastic model for PTA1 experiment

sensors 22-1 and 22-2 when neglecting hydration from the host rock along the radial boundary. However, the final relative humidity values are underestimated because the model does not consider the forced hydration from the end

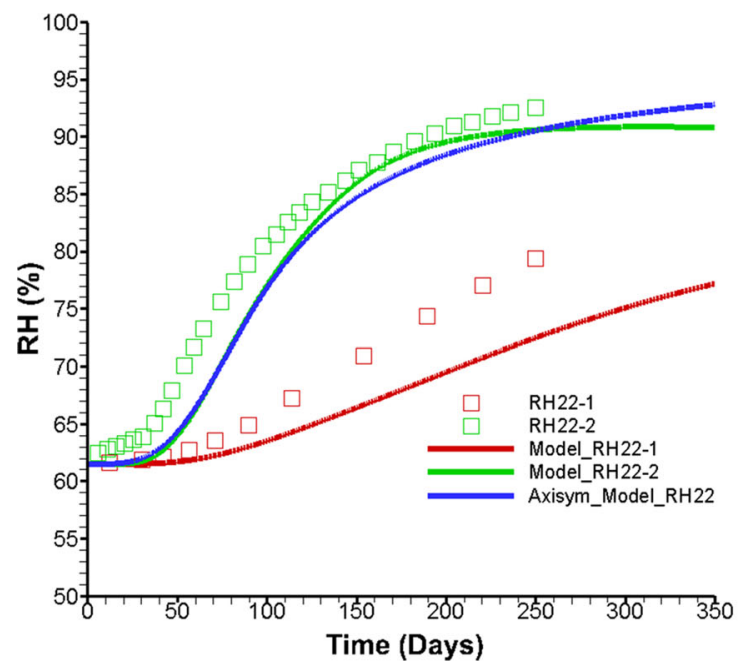

Fig. 21 Relative humidity data modelled at RH22 and RH52 sensors of the sample, as shown in Fig. 21. This is because the sensors RH22-1 and RH22-2 are located closer to the source of the forced hydration than RH52. The axisymmetric model suggests the hydrating front from the base reaches $0.22 \mathrm{~m}$ along the sample within a time frame of 80 days, after which Fig. 21 shows the model results diverging from the data. The greater divergence of the results for RH22-1 compared to RH22-2 suggests that the non-symmetric technological void results in a more significant contribution of fluid from the end hydration at RH22-1. Model results in which hydration continued through the radial boundary were not able to recreate the final relative humidity or the dissymmetry between sensors RH22-1 and RH22-2. Therefore, the results indicate that fluid from the host rock does not contribute significantly to the hydration of the sample. These results highlight the relative contributions of hydration from the technological void and from the forced end hydration.

Radial stress at sensor S60-2 is calculated with the 2D slice model and provides a good match to the rate of stress increase in comparison with experimental data (Fig. 22). However, the model calculates the technological void to be fully closed after 126 days and radial stress increases from this point. Similarly to the Step 1 model results, the model

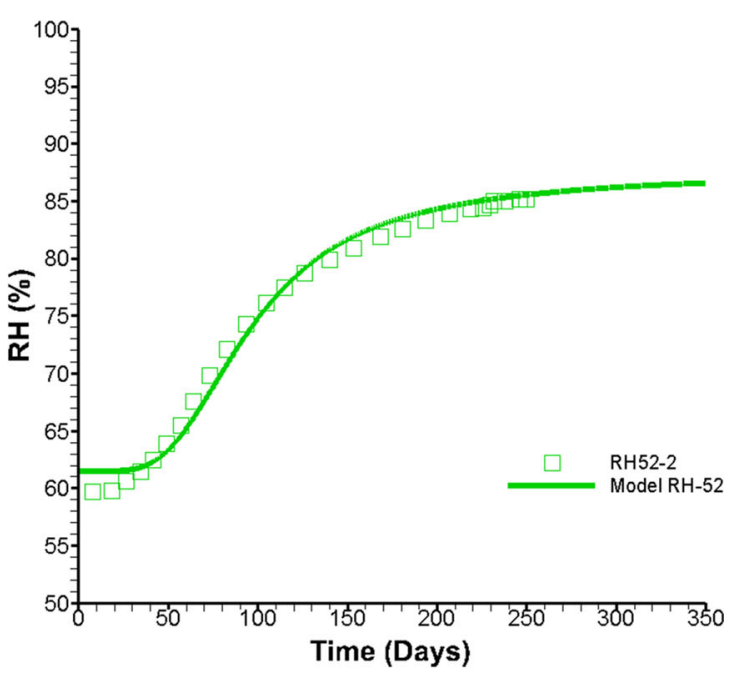




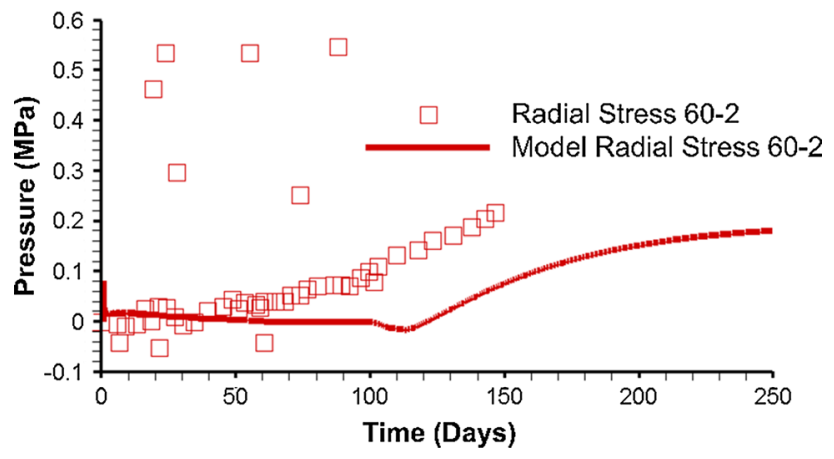

Fig. 22 Radial stress calculated at the top of the sample (i.e. the largest technological void) for the 2D slice nonlinear elastic model

calculates a closure of the technological void later than observed in the experiment (40 days). Although the model does not capture the gradual increase in radial stress, which is likely due to the formation of mechanically weak gel in the technological void as observed in small-scale experiments (Saba et al. 2014b), the later development of stress is well matched.

\section{Conclusions}

The aim of this study was to investigate the complex hydro-mechanical behaviour of bentonite with as simple a constitutive model and as few parameters as possible. In order to do so, a nonlinear elastic model was coupled to an unsaturated fluid flow simulation and used to model the hydro-mechanical behaviour of compacted MX-80 bentonite in the SEALEX experiments. An Updated Lagrangian mesh application was implemented in OpenGeoSys and used to inform the material non-linearity in the mechanical solution.

A series of parameterisation laboratory tests were reproduced by the simple HM-coupled model with an adequate fit achieved for consolidation at different suctions and constant volume infiltration. The coupling mechanism of Dueck suction using the free swell water retention curve and mean net stress to determine constant volume water retention properties was shown to be suitable for modelling the water retention behaviour at different void ratios and stresses. This model was then extended to simulate the complex behaviour of bentonite swelling into a technological void in both small-scale laboratory experiments and a full-scale in situ performance test.

The technological void was modelled using displacement-dependent boundary conditions for the hydro-mechanical conditions of the gap without considering the formation of a colloid or gel. The implemented Updated Lagrangian mesh was used to determine the closure of the technological void. Calculated water uptake from the technological void indicates that the void is filled within 2 days.

The simple model requires few parameters to describe the hydro-mechanical behaviour of the simple laboratory tests and the full-scale in situ performance test PT-A1. However, the wetting-induced collapse hydro-mechanical phenomenon seen in the 1:10 scale mock-up test cannot be modelled using only a nonlinear elastic formulation. Instead, an extension to the model incorporating a source term to account for plastic strains is presented. This enables the main features of the experimental results, such as axial stress development, water uptake, and relative humidity in the different experimental conditions to be reproduced with just nine mechanical parameters.

This empirical model is able to simulate the reduction in axial stress during wetting-induced collapse, but the elastic assumption in the constitutive framework results in an over prediction of the rebound displacement upon loss of confinement that could potentially be better predicted with more complex elasto-plastic models.

Model simulations of the PT-A1 performance test reproduced the axial stress evolution at one end of the sample and non-symmetric water up take due to the geometry of the technological void. Furthermore, relative humidity results indicate that hydration of the hydraulic seal from the host rock is negligible.

Acknowledgements This work was part-funded by Radioactive Waste Management Limited (a wholly owned subsidiary of the UK Nuclear Decommissioning Authority) via a subcontract through Amec Foster Wheeler and Quintessa Ltd. The work described in this paper was conducted within the context of Task A of the international DECOVALEX project. The authors are grateful to the funding organisations who supported the work. The views expressed in the paper are, however, those of the authors and are not necessarily those of the funding organisations.

Open Access This article is distributed under the terms of the Creative Commons Attribution 4.0 International License (http://creative commons.org/licenses/by/4.0/), which permits unrestricted use, distribution, and reproduction in any medium, provided you give appropriate credit to the original author(s) and the source, provide a link to the Creative Commons license, and indicate if changes were made.

\section{References}

Achari G, Joshi RC, Bentley RL, Chatterji S (1999) Prediction of the hydraulic conductivity of clays using the electric double layer theory. Can Geotech J 36(5):783-792

Agus SS (2005) An experimental study on hydrodynamic characteristics of compacted bentonite-sand mixtures. PhD Thesis, Bauhaus-University, Weimar, Germany

Agus SS, Schanz T, Fredlund DG (2010) Measurements of suction versus water content for bentonite-sand mixtures. Can Geotech J 47(5):583-594. doi:10.1139/T09-120

Alonso EE, Josa A, Gens A (1990) A constitutive model for partially saturated soils. Géotechnique 40(3):405-430. doi:10.1680/geot. 1990.40.3.405 
Alonso EE, Vaunat J, Gens A (1999) Modelling the mechanical behaviour of expansive clays. Eng Geol 54(1-2):173-183. doi:10.1016/S0013-7952(99)00079-4

Alonso EE, Romero E, Hoffmann C (2011) Hydromechanical behaviour of compacted granular expansive mixtures: experimental and constitutive study. Géotechnique 61(4):329-344

Andra (2005) Evaluation of the feasability of a geological repository in an argillaceous formation: Muese/Haute-Marne site. Dossier 2005-Argile

Barnichon J, Deleruyelle F (2010) Sealing experiments at the Tournemire URL. Towards convergence of technical nuclear safety practices in Europe, EUROSAFE (2009)

Barnichon JD, Dick P, Bauer C (2012) The SEALEX in situ experiments: performance tests of repository seals, In: Harmonising Rock Engineering and the Environment. Taylor and Francis Group, London

Blatz JA, Cui Y-J, Oldecop L (2008) Vapour equilibrium and osmotic technique for suction control. Geotech Geol Eng 26(6):661-673. doi:10.1007/s10706-008-9196-1

Bond AE, Benbow SJ (2009) QPAC multi-phase flow module functional specification and architectural design. Quintessa report QRS-QPAC-HYD-2 v1.0

Bond A, Thatcher K, Chittenden N, McDermott C, Fraser-Harris A (2014a) RWM coupled processes project: first annual report for RWM participation in DECOVALEX-2015 tasks A and C1. AMEC report 18040-TR-002 v2.1

Bond A, Thatcher K, Chittenden N, McDermott C, Fraser-Harris A (2015a) RWM coupled processes project: second annual report for RWM participation in DECOVALEX-2015 tasks A and C1. AMEC report 18040-TR-003 v3.0

Bond A, Thatcher K, Chittenden N, McDermott C, Fraser-Harris A (2015b) RWM coupled processes project: third annual report for RWM participation in DECOVALEX-2015 tasks A and C1. AMEC Foster Wheeler report 18040-TR-004 v2.0

Bond A, Thatcher K, Chittenden N, McDermott C, Fraser-Harris A, Wilson J (2015c) Final report of the coupled processes project: outcomes from DECOVALEX-2015. AMEC Foster Wheeler report 18040-TR-005 v2.0

Börgesson L, Sanden T, Johannesson L-E, Hernelind J (1995) Modelling of the physical behaviour of water saturated clay barriers. Laboratory tests, material models and finite element application. SKB, September

Celia MA, Bouloutas ET, Zarba RL (1990) A general massconservative numerical solution for the unsaturated flow equation. Water Resour Res 26:1483-1496. doi:10.1029/90WR00196

Cui YJ, Delage P, Yahia-Aissa M (2002) A model for the volume change behavior of heavily compacted swelling clays. Eng Geol 64(2-3):233-250. doi:10.1016/S0013-7952(01)00113-2

Dueck A (2004) Hydro-mechanical properties of a water unsaturated sodium bentonite. Lund University, Lund

Dueck A, Börgesson L (2007) Model suggested for an important part of the hydro-mechanical behaviour of a water unsaturated bentonite. Eng Geol 92(3-4):160-169. doi:10.1016/j.enggeo. 2007.04.004

Ferrari A, Seiphoori A (2015) Cyclic water retention behaviour of compacted granular bentonite. In: EAGE, pp 13-15

Fredlund DG (2002) Use of soil-water characteristic curve in the implementation of unsaturated soil mechanics. In: Third international conference on unsaturated soils

Fredlund DG, Rahardjo H (1993) Soil mechanics for unsaturated soils. Wiley, New York

Fredlund DG, Rahardjo H, Fredlund MD (2012) Unsaturated soil mechanics in engineering practice. Wiley, New York

Gallipoli D, Wheeler SJ, Karstunen M (2003) Modelling the variation of the degree of saturation in a deformable unsaturated soil. Geotechnique 53:105-112
Gatabin C, Talandier J, Collin F, Charlier R, Dieudonne A-C (2016) Competing effects of volume change and water uptake on the water retention behaviour of a compacted MX-80 bentonite/sand mixture. Appl Clay Sci 121-122:57-62

Gens A, Alonso EE (1992) A framework for the behaviour of unsaturated expansive clays. Can Geotech J 29:1013-1032

Gens A, Sánchez M, Sheng D (2006) On constitutive modelling of unsaturated soils. Acta Geotech 1(3):137-147. doi:10.1007/ s11440-006-0013-9

Gens A, Sánchez M, Valleján B, Imbert C, Villar MV, Van Geet M (2011) Hydromechanical behaviour of a heterogeneous compacted soil: experimental observations and modelling. Géotechnique 61(5):367-386. doi:10.1680/geot.SIP11.P.015

Hansen J, Holt E, Palmu M (2013) Full-scale demonstration of plugs and seals. In: Euradwaste'13 8th EC conference on the management of radioactive waste, community policy and research on disposal, Vilnius, Lithuania

Karnland O, Olsson S, Nilsson U (2006) Mineralogy and sealing properties of various bentonites and smectite-rich clay materials. SKB Technical report, TR-06-30

Kim J-S, Kwon S-K, Sanchez M, Cho G-C (2011) Geological storage of high level nuclear waste. KSCE J Civ Eng 15(4):721-737. doi:10.1007/s12205-011-0012-8

Kolditz O, Görke U-J, Shao H, Wang W, Bauer S (eds) (2016) Thermo-hydro-mechanical-chemical processes in fractured porous media: modelling and benchmarking-benchmarking initiatives. Springer. ISBN 978-3-319-29224-3

Kröhn K-P (2003) New conceptual models for the resaturation of bentonite. Appl Clay Sci 23(1-4):25-33. doi:10.1016/S01691317(03)00083-8

Laloui L, Nuth M (2005) An introduction to the constitutive modelling of unsaturated soils. Rev Eur Génie Civ 9(5-6):651-669. doi:10.1080/17747120.2005.9692775

Lewis RW, Schrefler BA (1998) The finite element method in the static and dynamic deformation and consolidation of porous media. Wiley, New York

Likos WJ, Lu N (2006) Pore-scale analysis of bulk volume change from crystalline interlayer swelling in $\mathrm{Na}^{+}$and $\mathrm{Ca}^{2+}$ smectite. Clays Clay Miner 54(4):516-529. doi:10.1346/CCMN.2006. 0540412

Liu L (2010) Permeability and expansibility of sodium bentonite in dilute solutions. Colloids Surf A 358(1-3):68-78. doi:10.1016/j. colsurfa.2010.01.033

Liu L, Neretnieks I, Moreno L (2011) Permeability and expansibility of natural bentonite MX-80 in distilled water. Phys Chem Earth Parts A/B/C 36(17-18):1783-1791. doi:10.1016/j.pce.2011.07. 009

Lloret A, Villar MV, Alonso EE, Sánchez M, Gens A, Pintado X (2003) Mechanical behaviour of heavily compacted bentonite under high suction changes. Géotechnique 53(1):27-40. doi:10. 1680/geot.2003.53.1.27

Madsen FT, Müller-Vonmoos M (1989) The swelling behaviour of clays. Appl Clay Sci 4(2):143-156. doi:10.1016/01691317(89)90005-7

Millard A, Mokni N, Barnichon JD, Thatcher KE, Bond AE, FraserHarris A, Mc Dermott C, Blaheta R, Michalec Z, Hasal M, Nguyen TS, Nasir O, Fedors R, Yi H, Kolditz O (2016) Comparative modelling of laboratory experiments for the hydromechanical behaviour of compacted bentonite-sand mixture. http://www.decovalex.org/resources.html\#special-issues

NAGRA (2009) The Nagra Research, Development and Demonstration (RD\&D) Plan for the Disposal of Radioactive Waste in Switzerland. Technical report 09-06

Navarro V, Asensio L, Yustres Á, Pintado X, Alonso J (2014) An elastoplastic model of bentonite free swelling. Eng Geol 181:190-201. doi:10.1016/j.enggeo.2014.07.014 
Navarro V, Asensio L, De la Morena G, Pintado X, Yustres Á (2015) Differentiated intra- and inter-aggregate water content models of MX-80 bentonite. Appl Clay Sci. doi:10.1016/j.clay.2015.10.015

Nguyen TSS, Selvadurai APS, Armand G, Selvadurai APS (2005) Modelling the FEBEX THM experiment using a state surface approach. Int J Rock Mech Miner Sci 42(5-6):639-651. doi:10. 1016/j.ijrmms.2005.03.005

Nuth M, Laloui L (2008) Effective stress concept in unsaturated soils: clarification and validation of a unified framework. J Numer Anal Methods. doi:10.1002/nag

Oy P (2010) Interim summary report of the safety case interim. Summary report of the safety case

Pusch R, Yong RN (2003) Water saturation and retention of hydrophylic clay buffer-microstrcutural aspects. Appl Clay Sci 23:61-68

Pusch R, Yong RN (2006) Microstructure of smectite clays and engineering performance. Taylor and Francis, London

Romero E, Gens A, Lloret A (2003) Suction effects on a compacted clay under non-isothermal conditions. Géotechnique 53(1):65-81. doi:10.1680/geot.53.1.65.37254

Rutqvist J, Ijiri Y, Yamamoto Y (2011) Implementation of the Barcelona Basic Model into TOUGH-FLAC for simulations of the geomechanical behaviour of unsaturated soils. Comput Geosci 37:751-762

Saba S, Barnichon J-D, Cui Y-J, Tang AM, Delage P (2014a) Microstructure and anisotropic swelling behaviour of compacted bentonite/sand mixture. J Rock Mech Geotech Eng 6(2):126-132. doi:10.1016/j.jrmge.2014.01.006

Saba S, Romero E, Barnichon J, Delage P (2014b) Hydro-mechanical behaviour of bentonite-sand mixture used as sealing materials in radioactive waste disposal galleries. Docteur de l' Université Paris-Est Comportement hydromécanique différé des barrières ouvragées argileuses gonflantes
Sánchez M, Gens A, do Nascimento Guimarães L, Olivella S (2005) A double structure generalized plasticity model for expansive materials. Int J Numer Anal Methods Geomech 29(8):751-787. doi:10.1002/nag.434

Thorenz C (2001) Model adaptive simulation of multiphase and density driven flow in fractured and porous media. PhD Thesis, Institute of Fluid Mechanics, Hannover University, Germany

van Genuchten MT (1980) A closed-from equation for predicting the hydraulic conductivity of unsaturated soils. Soil Sci Soc Am J 44:892-898

Wang Q (2012) Hydro-mechanical behaviour of bentonite-based materials used for high-level radioactive waste disposal, Phd Thesis. Ecole des Pont, Paristech, Paris, France

Wang Q, Tang AM, Cui Y-J, Delage P, Gatmiri B (2012) Experimental study on the swelling behaviour of bentonite/claystone mixture. Eng Geol 124:59-66. doi:10.1016/j.enggeo.2011.10.003

Wang Q, Cui Y-J, Minh Tang A, Xiang-Ling L, Wei-Min Y (2014) Time- and density-dependent microstructure features of compacted bentonite. Soils Found 54(4):657-666. doi:10.1016/j. sandf.2014.06.021

Wayllace A (2008) Volume change and swelling pressure of expansive clay in the crystalline swelling regime. $\mathrm{PhD}$ thesis, University of Missouri, USA

Wheeler SJ, Sivakumar V (1995) An elasto-plastic critical state framework for unsaturated soil. Géotechnique 45(1):35-53. doi:10.1680/geot.1995.45.1.35

Ye WMM, Cui YJJ, Qian LXX, Chen B (2009) An experimental study of the water transfer through confined compacted GMZ bentonite. Eng Geol 108(3-4):169-176. doi:10.1016/j.enggeo. 2009.08.003

Zhou A, Sheng D (2015) An advanced hydro-mechanical constitutive model for unsaturated soils with different initial densities. Comput Geotech 63:46-66. doi:10.1016/j.compgeo.2014.07.017 